\title{
A Wave Equation including Leptons and Quarks for the Standard Model of Quantum Physics in Clifford Algebra
}

\author{
Claude Daviau, Jacques Bertrand \\ Le Moulin de la Lande, Pouillé-les-Coteaux, France \\ Email: Claude.Daviau@nordnet.fr, bertrandjacques-m@orange.fr
}

Received 31 October 2014; revised 27 November 2014; accepted 21 December 2014

Copyright (C) 2014 by authors and Scientific Research Publishing Inc.

This work is licensed under the Creative Commons Attribution International License (CC BY). http://creativecommons.org/licenses/by/4.0/

\section{(c) (i) Open Access}

\begin{abstract}
A wave equation with mass term is studied for all fermionic particles and antiparticles of the first generation: electron and its neutrino, positron and antineutrino, quarks $u$ and $d$ with three states of color and antiquarks $\bar{u}$ and $\bar{d}$. This wave equation is form invariant under the $\mathrm{Cl}_{3}^{*}$ group generalizing the relativistic invariance. It is gauge invariant under the $U(1) \times S U(2) \times S U(3)$ group of the standard model of quantum physics. The wave is a function of space and time with value in the Clifford algebra $\mathrm{Cl}_{1,5}$. Then many features of the standard model, charge conjugation, color, left waves, and Lagrangian formalism, are obtained in the frame of the first quantization.
\end{abstract}

\section{Keywords}

Invariance Group, Dirac Equation, Electromagnetism, Weak Interactions, Strong Interactions, Clifford Algebras

\section{Introduction}

We use here all notations of "new insights in the standard model of quantum physics in Clifford algebra” [1]. The wave equation for all particles of the first generation is a generalization of the wave equation obtained in 6.7 for the electron and its neutrino. This wave equation has obtained a proper mass term compatible with the gauge invariance in [2]. It is a generalization of the homogeneous nonlinear Dirac equation for the electron alone [3]-[9]. 


$$
\nabla \hat{\phi}+q A \hat{\phi} \sigma_{12}+m \mathrm{e}^{-i \beta} \phi \sigma_{12}=0, \quad q=\frac{e}{\hbar c}, \quad m=\frac{m_{0} c}{\hbar}
$$

with

$$
\begin{aligned}
& \phi_{e}=\sqrt{2}\left(\begin{array}{cc}
\xi_{1 e} & -\eta_{2 e}^{*} \\
\xi_{2 e} & \eta_{1 e}^{*}
\end{array}\right)=\sqrt{2}\left(\xi_{e}-i \sigma_{2} \eta_{e}^{*}\right) \\
& \hat{\phi}_{e}=\sqrt{2}\left(\begin{array}{cc}
\eta_{1 e} & -\xi_{2 e}^{*} \\
\eta_{2 e} & \xi_{1 e}^{*}
\end{array}\right)=\sqrt{2}\left(\eta_{e}-i \sigma_{2} \xi_{e}^{*}\right) .
\end{aligned}
$$

Here $\xi_{e}$ and $\eta_{e}$ are respectively the right and left Weyl spinors of the electron. The $\beta$ angle is the YvonTakabayasi angle satisfying

$$
\operatorname{det}(\phi)=\Omega_{1}+i \Omega_{2}=\rho \mathrm{e}^{i \beta} .
$$

The link with the usual presentation of the standard model is made by the left and right Weyl spinors used for waves of each particle. These right and left waves are parts of the wave with value in $\mathrm{Cl}_{1,5}$.

We used previously the same algebra $C l_{5,1}=C l_{1,5}$. It is the same algebra, and this explains very well why sub-algebras $C l_{1,3}$ and $C l_{3,1}$ have been equally used to describe relativistic physics [10] [11]. But the signature of the scalar product cannot be free, this scalar product being linked to the gravitation in the general relativity. It happens that vectors of $C l_{1,5}$ are pseudo-vectors of $C l_{5,1}$ and more generally that $n$-vectors of $\mathrm{Cl}_{1,5}$ are $(6-n)$-vectors of $\mathrm{Cl}_{5,1}$. The generalization of the wave equation for electron-neutrino is simpler if we use $\mathrm{Cl}_{1,5}$. This is the first indication that the signature +----- is the true one. We explain in Appendix A how the reverse in $C l_{1,5}$ is linked to the reverse in $C l_{1,3}$, a necessary condition to get the wave equation of all particles of the first generation.

We have noticed, for the electron alone firstly (see [8] 2.4), next for electron + neutrino [2] the double link existing between the wave equation and the Lagrangian density: It is well known that the wave equation may be obtained from the Lagrangian density by the variational calculus. The new link is that the real part of the invariant wave equation is simply $\mathcal{L}=0$. The Lagrangian formalism is then necessary, being a consequence of the wave equation. Next we have extended the double link to electro-weak interactions in the leptonic case (electron + neutrino). Now we are extending the double link to the gauge group of the standard model. The Lagrangian density must then be the real part of the invariant wave equation.

Moreover we generalized the non-linear homogeneous wave equation of the electron, and we got a wave equation with mass term [2], form invariant under the $C l_{3}^{*}=G L(2, \mathbb{C})$ group and gauge invariant under the $U(1) \times S U(2)$ gauge group of electro-weak interactions. Our aim is to explain how this may be extended to a wave equation with mass term, both form invariant under $\mathrm{Cl}_{3}^{*}$ and gauge invariant under the $U(1) \times S U(2) \times S U(3)$ gauge group of the standard model, including both electro-weak and strong interactions.

\section{From the Lepton Case to the Full Wave}

The standard model adds to the leptons (electron $e$ and its neutrino $n$ ) in the "first generation" two quarks $u$ and $d$ with three colour states each. Weak interactions acting only on left waves of quarks (and right waves of antiquarks) we write the wave of all fermions of the first generation as follows:

$$
\begin{aligned}
& \Psi=\left(\begin{array}{ll}
\Psi_{l} & \Psi_{r} \\
\Psi_{g} & \Psi_{b}
\end{array}\right), \quad \Psi_{l}=\left(\begin{array}{cc}
\phi_{e} & \phi_{n} \\
\hat{\phi}_{n} & \hat{\phi}_{e}
\end{array}\right)=\left(\begin{array}{cc}
\phi_{e} & \phi_{n} \\
\hat{\phi}_{\bar{n}} \sigma_{1} & \hat{\phi}_{\bar{e}} \sigma_{1}
\end{array}\right) \\
& \Psi_{r}=\left(\begin{array}{ll}
\phi_{d r} & \phi_{u r} \\
\hat{\phi}_{u r} & \hat{\phi}_{d r}
\end{array}\right)=\left(\begin{array}{cc}
\phi_{d r} & \phi_{u r} \\
\hat{\phi}_{\overline{u r}} \sigma_{1} & \hat{\phi}_{\overline{d r}} \sigma_{1}
\end{array}\right), \quad \Psi_{g}=\left(\begin{array}{cc}
\phi_{d g} & \phi_{u g} \\
\hat{\phi}_{u g} & \hat{\phi}_{d g}
\end{array}\right)=\left(\begin{array}{cc}
\phi_{d g} & \phi_{u g} \\
\hat{\phi}_{\overline{u g}} \sigma_{1} & \hat{\phi}_{\overline{d g}} \sigma_{1}
\end{array}\right), \\
& \Psi_{b}=\left(\begin{array}{ll}
\phi_{d b} & \phi_{u b} \\
\hat{\phi}_{u b} & \hat{\phi}_{d b}
\end{array}\right)=\left(\begin{array}{cc}
\phi_{d b} & \phi_{u b} \\
\hat{\phi}_{\bar{u} b} \sigma_{1} & \hat{\phi}_{\overline{d b}} \sigma_{1}
\end{array}\right) .
\end{aligned}
$$

The electro-weak theory [12] needs three spinorial waves in the electron-neutrino case: the right $\xi_{e}$ and the left $\eta_{e}$ of the electron and the left spinor $\eta_{n}$ of the electronic neutrino. The form invariance of the Dirac 
theory imposes to use $\phi_{e}$ for the electron and $\phi_{n}$ for its neutrino satisfying

$$
\begin{aligned}
& \phi_{e}=\sqrt{2}\left(\begin{array}{cc}
\xi_{1 e} & -\eta_{2 e}^{*} \\
\xi_{2 e} & \eta_{1 e}^{*}
\end{array}\right)=\sqrt{2}\left(\xi_{e}-i \sigma_{2} \eta_{e}^{*}\right), \quad \phi_{n}=\sqrt{2}\left(\begin{array}{cc}
0 & -\eta_{2 n}^{*} \\
0 & \eta_{1 n}^{*}
\end{array}\right) \\
& \hat{\phi}_{e}=\sqrt{2}\left(\begin{array}{cc}
\eta_{1 e} & -\xi_{2 e}^{*} \\
\eta_{2 e} & \xi_{1 e}^{*}
\end{array}\right)=\sqrt{2}\left(\eta_{e}-i \sigma_{2} \xi_{e}^{*}\right), \quad \hat{\phi}_{n}=\sqrt{2}\left(\begin{array}{ll}
\eta_{1 n} & 0 \\
\eta_{2 n} & 0
\end{array}\right)=\sqrt{2}\left(\begin{array}{ll}
\eta_{n} & 0
\end{array}\right) .
\end{aligned}
$$

Waves $\phi_{e}$ and $\phi_{n}$ are functions of space and time with value into the Clifford algebra $\mathrm{Cl}_{3}$ of the physical space. The standard model uses only a left $\eta_{n}$ wave for the neutrino. We always use the matrix representation (A.1) which allows to see the Clifford algebra $C l_{1,3}$ as a sub-algebra of $M_{4}(\mathbb{C})$. Under the dilation $R$ with ratio $r$ induced by any $M$ in $G L(2, \mathbb{C})$ we have (for more details, see [6]):

$$
\begin{aligned}
& x^{\prime}=M x M^{\dagger}, \quad \operatorname{det}(M)=r \mathrm{e}^{i \theta}, \quad x=x^{\mu} \sigma_{\mu}, \quad x^{\prime}=x^{\prime \mu} \sigma_{\mu} \\
& \xi^{\prime}=M \xi, \quad \eta^{\prime}=\hat{M} \eta, \quad \eta_{n}^{\prime}=\hat{M} \eta_{n}, \quad \phi_{e}^{\prime}=M \phi_{e}, \quad \phi_{n}^{\prime}=M \phi_{n} \\
& \Psi_{l}^{\prime}=\left(\begin{array}{ll}
\phi_{e}^{\prime} & \phi_{n}^{\prime} \\
\hat{\phi}_{n}^{\prime} & \hat{\phi}_{e}^{\prime}
\end{array}\right)=\left(\begin{array}{cc}
M & 0 \\
0 & \hat{M}
\end{array}\right)\left(\begin{array}{ll}
\phi_{e} & \phi_{n} \\
\hat{\phi}_{n} & \hat{\phi}_{e}
\end{array}\right)=N \Psi_{l} .
\end{aligned}
$$

The form (2.3) of the wave is compatible both with the form invariance of the Dirac theory and with the charge conjugation used in the standard model: the wave $\psi_{\bar{e}}$ of the positron satisfies

$$
\psi_{\bar{e}}=i \gamma_{2} \psi^{*} \Leftrightarrow \hat{\phi}_{\bar{e}}=\hat{\phi}_{e} \sigma_{1} \text {. }
$$

We can then think the $\Psi_{l}$ wave as containing the electron wave $\phi_{e}$, the neutrino wave $\phi_{n}$ and also the positron wave $\phi_{\bar{e}}$ and the antineutrino wave $\phi_{\bar{n}}$ :

$$
\Psi_{l}=\left(\begin{array}{cc}
\phi_{e} & \phi_{n} \\
\hat{\phi}_{\bar{n}} \sigma_{1} & \hat{\phi}_{\bar{e}} \sigma_{1}
\end{array}\right), \quad \phi_{\bar{e}}=\sqrt{2}\left(\begin{array}{cc}
\xi_{1 \bar{e}} & -\eta_{2 \bar{e}}^{*} \\
\xi_{2 \bar{e}} & \eta_{1 \bar{e}}^{*}
\end{array}\right), \quad \phi_{\bar{n}}=\sqrt{2}\left(\begin{array}{cc}
\xi_{1 \bar{n}} & 0 \\
\xi_{2 \bar{n}} & 0
\end{array}\right) .
$$

And the antineutrino has only a right wave. The multivector $\Psi_{l}(x)$ is usually an invertible element of the space-time algebra because (see [1] (6.250)) with:

$$
\begin{aligned}
& a_{1}=\operatorname{det}\left(\phi_{e}\right)=\phi_{e} \bar{\phi}_{e}=2\left(\xi_{1 e} \eta_{1 e}^{*}+\xi_{2 e} \eta_{2 e}^{*}\right) \\
& a_{2}=2\left(\xi_{1 e} \eta_{1 n}^{*}+\xi_{2 e} \eta_{2 n}^{*}\right)=2\left(\eta_{2 e}^{*} \eta_{1 n}^{*}-\eta_{1 e}^{*} \eta_{2 n}^{*}\right) \\
& a_{3}=2\left(\xi_{1 e} \eta_{1 n}^{*}+\xi_{2 e} \eta_{2 n}^{*}\right)
\end{aligned}
$$

we got

$$
\operatorname{det}\left(\Psi_{l}\right)=a_{1} a_{1}^{*}+a_{2} a_{2}^{*} .
$$

Most of the preceding presentation is easily extended to quarks. For each color $c=r, g, b$ the electroweak theory needs only left waves:

$$
\Psi_{c}=\left(\begin{array}{ll}
\phi_{d c} & \phi_{u c} \\
\hat{\phi}_{u c} & \hat{\phi}_{d c}
\end{array}\right), \quad \hat{\phi}_{d c}=\sqrt{2}\left(\begin{array}{ll}
\eta_{1 d c} & 0 \\
\eta_{2 d c} & 0
\end{array}\right), \quad \hat{\phi}_{u c}=\sqrt{2}\left(\begin{array}{ll}
\eta_{1 u c} & 0 \\
\eta_{2 u c} & 0
\end{array}\right) .
$$

The $\Psi$ wave is now a function of space and time with value into $C l_{1,5}=C l_{5,1}$ which is a sub-algebra (on the real field) of $\mathrm{Cl}_{5,2}=M_{8}(\mathbb{C})$ :

$$
\Psi=\left(\begin{array}{ll}
\Psi_{l} & \Psi_{r} \\
\Psi_{g} & \Psi_{b}
\end{array}\right), \quad \tilde{\Psi}=\left(\begin{array}{cc}
\tilde{\Psi}_{b} & \tilde{\Psi}_{r} \\
\tilde{\Psi}_{g} & \tilde{\Psi}_{l}
\end{array}\right)
$$

The link between the reverse in $\mathrm{Cl}_{1,5}$ and the reverse in $\mathrm{Cl}_{1,3}$ is not trivial and is detailed in Appendix A. The wave equation for all objects of the first generation reads

$$
0=(\underline{D} \Psi) L_{012}+\underline{\mathbf{M}} .
$$


The mass term reads

$$
\underline{\mathbf{M}}=\left(\begin{array}{cc}
m_{2} \rho_{2} \chi_{b} & m_{2} \rho_{2} \chi_{g} \\
m_{2} \rho_{2} \chi_{r} & m_{1} \rho_{1} \chi_{l}
\end{array}\right)
$$

where we use the scalar densities $s_{j}$ and $\chi$ terms of Appendix B, with

$$
\rho_{1}^{2}=a_{1} a_{1}^{*}+a_{2} a_{2}^{*}+a_{3} a_{3}^{*}, \quad \rho_{2}^{2}=\sum_{j=1}^{j=15} s_{j} s_{j}^{*} .
$$

The covariant derivative $\underline{D}$ uses the matrix representation (A.1) and reads

$$
\begin{aligned}
& \underline{D}=\underline{\partial}+\frac{g_{1}}{2} \underline{B} \underline{P}_{0}+\frac{g_{2}}{2} \underline{W}^{j} \underline{P}_{j}+\frac{g_{3}}{2} \underline{G}^{k} \underline{\mathbf{i}}_{k} \\
& \underline{D}=\sum_{\mu=0}^{3} L^{\mu} D_{\mu}, \quad \underline{\partial}=\sum_{\mu=0}^{3} L^{\mu} \partial_{\mu}, \quad \underline{B}=\sum_{\mu=0}^{3} L^{\mu} B_{\mu} \\
& \underline{W}^{j}=\sum_{\mu=0}^{3} L^{\mu} W_{\mu}^{j}, \quad j=1,2,3 \\
& \underline{G}^{k}=\sum_{\mu=0}^{3} L^{\mu} G_{\mu}^{k}, \quad k=1,2, \cdots, 8 .
\end{aligned}
$$

We use two projectors satisfying

$$
\underline{P}_{ \pm}(\Psi)=\frac{1}{2}\left(\Psi \pm \underline{\mathbf{i}} \Psi L_{21}\right), \quad \underline{\mathbf{i}}=L_{0123} .
$$

Three operators act on quarks like on leptons:

$$
\begin{aligned}
& \underline{P}_{1}(\Psi)=\underline{P}_{+}(\Psi) L_{35} \\
& \underline{P}_{2}(\Psi)=\underline{P}_{+}(\Psi) L_{0125} \\
& \underline{P}_{3}(\Psi)=\underline{P}_{+}(\Psi)(-\underline{\mathbf{i}}) .
\end{aligned}
$$

The fourth operator acts differently on the leptonic and on the quark sector. Using projectors:

$$
P^{+}=\frac{1}{2}\left(I_{8}+L_{012345}\right)=\left(\begin{array}{cc}
I_{4} & 0 \\
0 & 0
\end{array}\right), \quad P^{-}=\frac{1}{2}\left(I_{8}-L_{012345}\right)=\left(\begin{array}{cc}
0 & 0 \\
0 & I_{4}
\end{array}\right)
$$

we can separate the lepton part $\Psi^{l}$ and the quark part $\Psi^{c}$ of the wave:

$$
\Psi^{l}=P^{+} \Psi P^{+}=\left(\begin{array}{cc}
\Psi_{l} & 0 \\
0 & 0
\end{array}\right), \quad \Psi^{c}=\Psi-\Psi^{l}=\left(\begin{array}{cc}
0 & \Psi_{r} \\
\Psi_{g} & \Psi_{b}
\end{array}\right)
$$

and we get (see [1] (B.4) with $a=b=1$ )

$$
\begin{gathered}
\underline{P}_{0}\left(\Psi^{l}\right)=\frac{1}{2} \mathbf{i} \Psi^{l} L_{21}+\frac{3}{2} \Psi^{l} L_{21} \\
\underline{P}_{0}\left(\Psi^{c}\right)=-\frac{1}{3} \Psi^{c} L_{21} .
\end{gathered}
$$

This last relation comes from the non-existence of the right part of the $\Psi^{c}$ waves.

\section{Chromodynamics}

We start from generators $\lambda_{k}$ of the $S U(3)$ gauge group of chromodynamics 


$$
\begin{aligned}
& \lambda_{1}=\left(\begin{array}{lll}
0 & 1 & 0 \\
1 & 0 & 0 \\
0 & 0 & 0
\end{array}\right), \quad \lambda_{2}=\left(\begin{array}{ccc}
0 & -i & 0 \\
i & 0 & 0 \\
0 & 0 & 0
\end{array}\right), \quad \lambda_{3}=\left(\begin{array}{ccc}
1 & 0 & 0 \\
0 & -1 & 0 \\
0 & 0 & 0
\end{array}\right), \quad \lambda_{4}=\left(\begin{array}{lll}
0 & 0 & 1 \\
0 & 0 & 0 \\
1 & 0 & 0
\end{array}\right), \\
& \lambda_{5}=\left(\begin{array}{ccc}
0 & 0 & -i \\
0 & 0 & 0 \\
i & 0 & 0
\end{array}\right), \quad \lambda_{6}=\left(\begin{array}{ccc}
0 & 0 & 0 \\
0 & 0 & 1 \\
0 & 1 & 0
\end{array}\right), \quad \lambda_{7}=\left(\begin{array}{ccc}
0 & 0 & 0 \\
0 & 0 & -i \\
0 & i & 0
\end{array}\right), \quad \lambda_{8}=\frac{1}{\sqrt{3}}\left(\begin{array}{ccc}
1 & 0 & 0 \\
0 & 1 & 0 \\
0 & 0 & -2
\end{array}\right) \text {. }
\end{aligned}
$$

To simplify here notations we use now $l, r, g, b$ instead $\Psi_{l}, \Psi_{r}, \Psi_{g}, \Psi_{b}$. So we have $\Psi=\left(\begin{array}{ll}l & r \\ g & b\end{array}\right)$. Then (2.1) gives

$$
\begin{aligned}
& \lambda_{1}\left(\begin{array}{l}
r \\
g \\
b
\end{array}\right)=\left(\begin{array}{l}
g \\
r \\
0
\end{array}\right), \quad \lambda_{2}\left(\begin{array}{l}
r \\
g \\
b
\end{array}\right)=\left(\begin{array}{c}
-i g \\
i r \\
0
\end{array}\right), \quad \lambda_{3}\left(\begin{array}{l}
r \\
g \\
b
\end{array}\right)=\left(\begin{array}{c}
r \\
-g \\
0
\end{array}\right), \quad \lambda_{4}\left(\begin{array}{l}
r \\
g \\
b
\end{array}\right)=\left(\begin{array}{l}
b \\
0 \\
r
\end{array}\right), \\
& \lambda_{5}\left(\begin{array}{l}
r \\
g \\
b
\end{array}\right)=\left(\begin{array}{c}
-i b \\
0 \\
i r
\end{array}\right), \quad \lambda_{6}\left(\begin{array}{l}
r \\
g \\
b
\end{array}\right)=\left(\begin{array}{l}
0 \\
b \\
g
\end{array}\right), \quad \lambda_{7}\left(\begin{array}{l}
r \\
g \\
b
\end{array}\right)=\left(\begin{array}{c}
0 \\
-i b \\
i g
\end{array}\right), \quad \lambda_{8}\left(\begin{array}{l}
r \\
g \\
b
\end{array}\right)=\frac{1}{\sqrt{3}}\left(\begin{array}{c}
r \\
g \\
-2 b
\end{array}\right) .
\end{aligned}
$$

We name $\Gamma_{k}$ operators corresponding to $\lambda_{k}$ acting on $\Psi$. We get with projectors $P^{+}$and $P^{-}$in (2.27):

$$
\begin{aligned}
& \Gamma_{1}(\Psi)=\frac{1}{2}\left(L_{4} \Psi L_{4}+L_{01235} \Psi L_{01235}\right)=\left(\begin{array}{cc}
0 & g \\
r & 0
\end{array}\right) \\
& \Gamma_{2}(\Psi)=\frac{1}{2}\left(L_{5} \Psi L_{4}-L_{01234} \Psi L_{01235}\right)=\left(\begin{array}{cc}
0 & -\mathbf{i} g \\
\mathbf{i} r & 0
\end{array}\right) \\
& \Gamma_{3}(\Psi)=P^{+} \Psi P^{-}-P^{-} \Psi P^{+}=\left(\begin{array}{cc}
0 & r \\
-g & 0
\end{array}\right) \\
& \Gamma_{4}(\Psi)=L_{01253} \Psi P^{-}=\left(\begin{array}{ll}
0 & b \\
0 & r
\end{array}\right), \quad \Gamma_{5}(\Psi)=L_{01234} \Psi P^{-}=\left(\begin{array}{cc}
0 & -\mathbf{i} b \\
0 & \mathbf{i} r
\end{array}\right) \\
& \Gamma_{6}(\Psi)=P^{-} \Psi L_{01253}=\left(\begin{array}{ll}
0 & 0 \\
b & g
\end{array}\right), \quad \Gamma_{7}(\Psi)=-\underline{\mathbf{i}} P^{-} \Psi L_{4}=\left(\begin{array}{cc}
0 & 0 \\
-\mathbf{i} b & \mathbf{i} g
\end{array}\right) \\
& \Gamma_{8}(\Psi)=\frac{1}{\sqrt{3}}\left(P^{-} \Psi L_{012345}+L_{012345} \Psi P^{-}\right)=\frac{1}{\sqrt{3}}\left(\begin{array}{cc}
0 & r \\
g & -2 b
\end{array}\right) .
\end{aligned}
$$

Everywhere the left up term is 0 , so all $\Gamma_{k}$ project the wave $\Psi$ on its quark sector.

We can extend the covariant derivative of electro-weak interactions in the electron-neutrino case:

$$
D \Psi_{l}=\partial \Psi_{l}+\frac{g_{1}}{2} B P_{0}\left(\Psi_{l}\right)+\frac{g_{2}}{2} W^{j} P_{j}\left(\Psi_{l}\right)
$$

to get the covariant derivative of the standard model

$$
\underline{D}(\Psi)=\underline{\partial}(\Psi)+\frac{g_{1}}{2} \underline{B P}_{0}(\Psi)+\frac{g_{2}}{2} \underline{W}^{j} \underline{P}_{j}(\Psi)+\frac{g_{3}}{2} \underline{G}^{k} \underline{\mathbf{i}} \Gamma_{k}(\Psi)
$$

where $g_{3}$ is another constant and $\underline{G}^{k}$ are eight terms called "gluons". Since $I_{4}$ commute with any element of $C l_{1,3}$ and since $P_{\mu}\left(\mathbf{i} \Psi_{\text {ind }}\right)=\mathbf{i} P_{\mu}\left(\Psi_{\text {ind }}\right)$ for $\mu=0,1,2,3$ and ind $=l, r, g, b$ each operator $\underline{\mathbf{i}} \Gamma_{k}$ commutes with all operators $\underline{P}_{j}$.

Now we use 12 real numbers $a^{0}, a^{j}, j=1,2,3, b^{k}, k=1,2, \cdots, 8$, we let

$$
S_{0}=a^{0} \underline{P}_{-0}, \quad S_{1}=\sum_{j=1}^{j=3} a^{j} \underline{P}_{j}, \quad S_{2}=\sum_{k=1}^{k=8} b^{k} \underline{\mathbf{i}} \Gamma_{k}, \quad S=S_{0}+S_{1}+S_{2}
$$


and we get, using exponentiation

$$
\exp (S)=\exp \left(S_{0}\right) \exp \left(S_{1}\right) \exp \left(S_{2}\right)=\exp \left(S_{1}\right) \exp \left(S_{0}\right) \exp \left(S_{2}\right)=\exp \left(S_{0}\right) \exp \left(S_{2}\right) \exp \left(S_{1}\right)=\cdots
$$

in any order. The set of these operators $\exp (S)$ is a $U(1) \times S U(2) \times S U(3)$ Lie group. Only difference with the standard model: the structure of this group is not postulated but calculated. With

$$
\Psi^{\prime}=[\exp (S)](\Psi), \quad \underline{D}=L^{\mu} \underline{D}_{\mu}, \quad \underline{D}^{\prime}=L^{\mu} \underline{D}_{\mu}^{\prime}
$$

the gauge transformation reads

$$
\begin{aligned}
\underline{D}_{\mu}^{\prime} \Psi^{\prime} & =[\exp (S)]\left(\underline{D}_{\mu} \Psi\right) \\
B_{\mu}^{\prime}=B_{\mu} & -\frac{2}{g_{1}} \partial_{\mu} a^{0} \\
W_{\mu}^{\prime j} \underline{P}_{j} & =\left[\exp \left[S_{1}\right] W_{\mu}^{j} \underline{P}_{j}-\frac{2}{g_{2}} \partial_{\mu}\left[\exp \left(S_{1}\right)\right]\right] \exp \left(-S_{1}\right) \\
\underline{G}_{\mu}^{\prime k} \underline{\mathbf{i}} \Gamma_{k} & =\left[\exp \left(S_{2}\right) \underline{G}_{\mu}^{k} \mathbf{i} \Gamma_{k}-\frac{2}{g_{3}} \partial_{\mu}\left[\exp \left(S_{2}\right)\right]\right] \exp \left(-S_{2}\right) .
\end{aligned}
$$

The $S U(3)$ group generated by operators $\Gamma_{k}$ acts only on the quark sector of the wave:

$$
P^{+}\left[\exp \left(b^{k} \underline{\mathbf{i}} \Gamma_{k}\right)\right](\Psi) P^{+}=P^{+} \Psi P^{+}=\Psi^{l} .
$$

The physical translation is: Leptons do not act by strong interactions. This comes from the structure of the wave itself. It is fully satisfied in experiments. We get then a $U(1) \times S U(2) \times S U(3)$ gauge group for a wave including all fermions of the first generation. This group acts on the lepton sector only by its $U(1) \times S U(2)$ part. Consequently the wave equation is composed of a lepton wave equation and a quark wave equation:

$$
\begin{aligned}
& 0=\left(\underline{D} \Psi^{l}\right) L_{012}+m_{1} \rho_{1}\left(\begin{array}{cc}
0 & 0 \\
0 & \chi_{l}
\end{array}\right) \\
& 0=\left(\underline{D}^{c}\right) L_{012}+m_{2} \rho_{2} \chi^{c}, \quad \chi^{c}=\left(\begin{array}{cc}
\chi_{b} & \chi_{g} \\
\chi_{r} & 0
\end{array}\right) .
\end{aligned}
$$

The wave equation (3.19) is equivalent to the wave equation

$$
\mathbf{D} \Psi_{1} \gamma_{012}+m_{1} \rho_{1} \chi_{1}=0, \quad \gamma_{012}=\gamma_{0} \gamma_{1} \gamma_{2}
$$

studied in [2] [13], where

$$
\begin{aligned}
& \chi_{I}=\frac{1}{\rho_{1}^{2}}\left(\begin{array}{cc}
a_{1}^{*} \phi_{e}+a_{2}^{*} \phi_{n} \sigma_{1}+a_{3}^{*} \phi_{n} & -a_{2}^{*} \phi_{e L} \sigma_{1}+a_{3}^{*} \phi_{e R} \\
a_{2} \hat{\phi}_{e L} \sigma_{1}+a_{3} \hat{\phi}_{e R} & a_{1} \hat{\phi}_{e}-a_{2} \hat{\phi}_{n} \sigma_{1}+a_{3} \hat{\phi}_{n}
\end{array}\right) \\
& \phi_{e R}=\phi_{e} \frac{1+\sigma_{3}}{2}, \quad \phi_{e L}=\phi_{e} \frac{1-\sigma_{3}}{2} .
\end{aligned}
$$

This wave equation is equivalent to the invariant equation:

$$
\tilde{\Psi}_{l}\left(\mathbf{D} \Psi_{l}\right) \gamma_{012}+m \rho_{1} \tilde{\Psi}_{l} \chi_{l}=0, \quad \tilde{\Psi}_{l}=\left(\begin{array}{ll}
\bar{\phi}_{e} & \varphi_{n}^{\dagger} \\
\bar{\phi}_{n} & \varphi_{e}^{\dagger}
\end{array}\right) .
$$

This wave equation is form invariant under the Lorentz dilation $R$ induced by any invertible matrix $M$ satisfying (2.5), (2.6), (2.7) [1]. It is gauge invariant under the $U(1) \times S U(2)$ group [2] generated by operators $P_{\mu}$ which are projections on the lepton sector of the operators defined in (2.23) to (2.29). Therefore we need to study only the quark sector and its wave equation (3.20).

We begin by the double link between wave equation and Lagrangian density that we have remarked firstly in the Dirac equation [8], next in the lepton case electron + neutrino [1]. 


\section{Double Link between Wave Equation and Lagrangian Density}

The existence of a Lagrangian mechanism in optics and mechanics is known since Fermat and Maupertuis. This principle of minimum is everywhere in quantum mechanics from its beginning, it is the main reason of the hypothesis of a wave linked to the move of any material particle made by L. de Broglie [14]. By the calculus of variations it is always possible to get the wave equation from the Lagrangian density. But another link exists: the Lagrangian density is the real scalar part of the invariant wave equation. This was obtained firstly for the electron alone [8], next for the pair electron-neutrino [2] where the Lagrangian density reads

$$
\begin{aligned}
& \mathcal{L}_{1}=\mathcal{L}_{0}+g_{1} \mathcal{L}_{1}+g_{2} \mathcal{L}_{2}+m_{1} \rho_{1} \\
& \mathcal{L}_{0}=\mathfrak{R}\left[-i\left(\eta_{e}^{\dagger} \sigma^{\mu} \partial_{\mu} \eta_{e}+\xi_{e}^{\dagger} \hat{\sigma}^{\mu} \partial_{\mu} \xi_{e}+\eta_{n}^{\dagger} \sigma^{\mu} \partial_{\mu} \eta_{n}\right)\right] \\
& \mathcal{L}_{1}=B_{\mu}\left(\frac{1}{2} \eta_{e}^{\dagger} \sigma^{\mu} \eta_{e}+\xi_{e}^{\dagger} \hat{\sigma}^{\mu} \xi_{e}+\frac{1}{2} \eta_{n}^{\dagger} \sigma^{\mu} \eta_{n}\right) \\
& \mathcal{L}_{2}=-\mathfrak{R}\left[\left(W_{\mu}^{1}+i W_{\mu}^{2}\right) \eta_{e}^{\dagger} \sigma^{\mu} \eta_{n}\right]+\frac{W_{\mu}^{3}}{2}\left(\eta_{e}^{\dagger} \sigma^{\mu} \eta_{e}-\eta_{n}^{\dagger} \sigma^{\mu} \eta_{n}\right)
\end{aligned}
$$

We shall establish the double link now for the wave equation (2.16). It is sufficient to add the property for (3.20). This equation is equivalent to the invariant equation:

$$
\begin{aligned}
& 0=\tilde{\Psi}^{c}\left(\underline{D}^{c}\right) L_{012}+m_{2} \rho_{2} \tilde{\Psi}^{c} \chi^{c} \\
& \tilde{\Psi}^{c}=\left(\begin{array}{cc}
\tilde{\Psi}_{b} & \tilde{\Psi}_{r} \\
\tilde{\Psi}_{g} & 0
\end{array}\right), \quad \chi^{c}=\left(\begin{array}{cc}
\chi_{b} & \chi_{g} \\
\chi_{r} & 0
\end{array}\right) .
\end{aligned}
$$

We get from the covariant derivative (2.19) with the operators $\underline{P}_{j}$ in (2.24), (2.25), (2.26) and (2.30) and $\Gamma_{k}$ in (3.3) to (3.8) and with $\Psi^{c}$ in (2.28)

$$
\begin{gathered}
\underline{D} \Psi^{c}=\left(\begin{array}{cc}
A_{g} & A_{b} \\
0 & A_{r}
\end{array}\right) \\
A_{g}=\partial \Psi_{g}-\frac{g_{1}}{6} \mathbf{B} \Psi_{g} \gamma_{21}+\frac{g_{2}}{2}\left(\mathbf{W}^{1} \Psi_{g} \gamma_{3} \mathbf{i}+\mathbf{W}^{2} \Psi_{g} \gamma_{3}-\mathbf{W}^{3} \Psi_{g} \mathbf{i}\right) \\
+\frac{g_{3}}{2}\left(\mathbf{G}^{1} \mathbf{i} \Psi_{r}-\mathbf{G}^{2} \Psi_{r}-\mathbf{G}^{3} \mathbf{i} \Psi_{g}+\mathbf{G}^{6} \mathbf{i} \Psi_{b}+\mathbf{G}^{7} \Psi_{b}+\frac{1}{\sqrt{3}} \mathbf{G}^{8} \mathbf{i} \Psi_{g}\right), \\
A_{b}=\partial \Psi_{b}-\frac{g_{1}}{6} \mathbf{B} \Psi_{b} \gamma_{21}+\frac{g_{2}}{2}\left(\mathbf{W}^{1} \Psi_{b} \gamma_{3} \mathbf{i}+\mathbf{W}^{2} \Psi_{b} \gamma_{3}-\mathbf{W}^{3} \Psi_{b} \mathbf{i}\right) \\
+\frac{g_{3}}{2}\left(\mathbf{G}^{4} \mathbf{i} \Psi_{r}-\mathbf{G}^{5} \Psi_{r}+\mathbf{G}^{6} \mathbf{i} \Psi_{g}-\mathbf{G}^{7} \Psi_{g}-\frac{2}{\sqrt{3}} \mathbf{G}^{8} \mathbf{i} \Psi_{b}\right) \\
A_{r}=\partial \Psi_{r}-\frac{g_{1}}{6} \mathbf{B} \Psi_{r} \gamma_{21}+\frac{g_{2}}{2}\left(\mathbf{W}^{1} \Psi_{r} \gamma_{3} \mathbf{i}+\mathbf{W}^{2} \Psi_{r} \gamma_{3}-\mathbf{W}^{3} \Psi_{r} \mathbf{i}\right) \\
+\frac{g_{3}}{2}\left(\mathbf{G}^{1} \mathbf{i} \Psi_{g}+\mathbf{G}^{2} \Psi_{g}+\mathbf{G}^{3} \mathbf{i} \Psi_{r}+\mathbf{G}^{4} \mathbf{i} \Psi_{b}+\mathbf{G}^{5} \Psi_{b}+\frac{1}{\sqrt{3}} \mathbf{G}^{8} \mathbf{i} \Psi_{r}\right)
\end{gathered}
$$

Next we get

$\tilde{\Psi}^{c}\left(\underline{D}^{c}\right) L_{012}+m_{2} \rho_{2} \tilde{\Psi}^{c} \chi^{c}=\left(\begin{array}{cc}\tilde{\Psi}_{b}\left(A_{b} \gamma_{012}+m_{2} \rho_{2} \chi_{b}\right)+\tilde{\Psi}_{r}\left(A_{r} \gamma_{012}+m_{2} \rho_{2} \chi_{r}\right) & \tilde{\Psi}_{b}\left(A_{g} \gamma_{012}+m_{2} \rho_{2} \chi_{g}\right) \\ \tilde{\Psi}_{g}\left(A_{b} \gamma_{012}+m_{2} \rho_{2} \chi_{b}\right) & \tilde{\Psi}_{g}\left(A_{g} \gamma_{012}+m_{2} \rho_{2} \chi_{g}\right)\end{array}\right)$

The calculation of the Lagrangian density in the general case is similar to the lepton case. We get

$$
\mathcal{L}=\mathcal{L}_{1}+\mathcal{L}_{c}
$$




$$
\mathcal{L}_{c}=\sum_{c=r, g, b} \mathcal{L}_{0 c}+g_{1} \sum_{c=r, g, b} \mathcal{L}_{1 c}+g_{2} \sum_{c=r, g, b} \mathcal{L}_{2 c}+g_{3} \mathcal{L}_{3}+m_{2} \rho_{2} .
$$

The calculation of $\mathcal{L}_{j c}, j=0,1,2$ replaces the pair $e-n$ by the pair $d c-u c$ and suppress the $\xi$ terms, then (4.2) (4.3) (4.4) become

$$
\begin{aligned}
& \mathcal{L}_{0 c}=\Re\left[-i\left(\eta_{d c}^{\dagger} \sigma^{\mu} \partial_{\mu} \eta_{d c}+\eta_{u c}^{\dagger} \sigma^{\mu} \partial_{\mu} \eta_{u c}\right)\right] \\
& \mathcal{L}_{1 c}=-\frac{B_{\mu}}{6}\left(\eta_{d c}^{\dagger} \sigma^{\mu} \eta_{d c}+\eta_{u c}^{\dagger} \sigma^{\mu} \eta_{u c}\right) \\
& \mathcal{L}_{2 c}=-\mathfrak{R}\left[\left(W_{\mu}^{1}+i W_{\mu}^{2}\right) \eta_{d c}^{\dagger} \sigma^{\mu} \eta_{u c}\right]+\frac{W_{\mu}^{3}}{2}\left(\eta_{d c}^{\dagger} \sigma^{\mu} \eta_{d c}-\eta_{u c}^{\dagger} \sigma^{\mu} \eta_{u c}\right)
\end{aligned}
$$

Since three $S U(2)$ group are included in $S U(3)$ the calculation of $\mathcal{L}_{3}$ has similarities with the calculation of $\mathcal{L}_{2}$ and we get

$$
\begin{aligned}
\mathcal{L}_{3}= & -\Re\left[\left(G_{\mu}^{1}+i G_{\mu}^{2}\right)\left(\eta_{d r}^{\dagger} \sigma^{\mu} \eta_{d g}+\eta_{u r}^{\dagger} \sigma^{\mu} \eta_{u g}\right)\right]-\mathfrak{R}\left[\left(G_{\mu}^{4}+i G_{\mu}^{5}\right)\left(\eta_{d r}^{\dagger} \sigma^{\mu} \eta_{d b}+\eta_{u r}^{\dagger} \sigma^{\mu} \eta_{u b}\right)\right] \\
& -\Re\left[\left(G_{\mu}^{6}+i G_{\mu}^{7}\right)\left(\eta_{d g}^{\dagger} \sigma^{\mu} \eta_{d b}+\eta_{u g}^{\dagger} \sigma^{\mu} \eta_{u b}\right)\right]+\frac{G_{\mu}^{3}}{2}\left(-\eta_{d r}^{\dagger} \sigma^{\mu} \eta_{d r}-\eta_{u r}^{\dagger} \sigma^{\mu} \eta_{u r}+\eta_{d g}^{\dagger} \sigma^{\mu} \eta_{d g}+\eta_{u g}^{\dagger} \sigma^{\mu} \eta_{u g}\right) \\
& +\frac{G_{\mu}^{8}}{2 \sqrt{3}}\left(-\eta_{d r}^{\dagger} \sigma^{\mu} \eta_{d r}-\eta_{u r}^{\dagger} \sigma^{\mu} \eta_{u r}+2 \eta_{d b}^{\dagger} \sigma^{\mu} \eta_{d b}+2 \eta_{u b}^{\dagger} \sigma^{\mu} \eta_{u b}-\eta_{d g}^{\dagger} \sigma^{\mu} \eta_{d g}-\eta_{u g}^{\dagger} \sigma^{\mu} \eta_{u g}\right)
\end{aligned}
$$

This new link between the wave equation and the Lagrangian density is much stronger than the old one, because it comes from a simple separation of the different parts of a multivector in Clifford algebra. The old link, going from the Lagrangian density to the wave equation, supposes a condition of cancellation at infinity which is dubious in the case of a propagating wave. On the physical point of view, there are no difficulties in the case of a stationary wave. Difficulties begin when propagating waves are studied. Our wave equations, since they are compatible with an oriented time and an oriented space, appear as more general, more physical, than Lagrangians. These are only particular consequences of the wave equations.

On the mathematical point of view the old link is always available. It is from the Lagrangian density (4.12) and using Lagrange equations that we have obtained the wave equation (2.16).

\section{Invariances}

\subsection{Form Invariance of the Wave Equation}

Under the Lorentz dilation $R$ induced by an invertible $M$ matrix satisfying

$$
\begin{aligned}
& x^{\prime}=M x M^{\dagger}, \quad \operatorname{det}(M)=r e^{i \theta}, \quad x=x^{\mu} \sigma_{\mu}, \quad x^{\prime}=x^{\prime \mu} \sigma_{\mu} \\
& \eta_{u c}^{\prime}=\hat{M} \eta_{u c}, \quad \eta_{d c}^{\prime}=\hat{M} \eta_{d c}, \quad \phi_{d c}^{\prime}=M \varphi_{d c}, \quad \phi_{u c}^{\prime}=M \varphi_{u c} \\
& \Psi_{c}^{\prime}=\left(\begin{array}{ll}
\phi_{d c}^{\prime} & \phi_{u c}^{\prime} \\
\hat{\phi}_{u c}^{\prime} & \hat{\phi}_{d c}^{\prime}
\end{array}\right)=\left(\begin{array}{cc}
M & 0 \\
0 & \hat{M}
\end{array}\right)\left(\begin{array}{ll}
\phi_{d c} & \phi_{u c} \\
\hat{\phi}_{u c} & \hat{\phi}_{d c}
\end{array}\right)=N \Psi_{c}, \quad c=r, g, b .
\end{aligned}
$$

We then let

which implies

$$
\underline{N}=\left(\begin{array}{cc}
N & 0 \\
0 & N
\end{array}\right), \quad \underline{\partial}=L^{\mu} \partial_{\mu}=\left(\begin{array}{ll}
0 & \partial \\
\partial & 0
\end{array}\right)
$$

$$
\Psi^{\prime c}=\underline{N} \Psi^{c}, \quad \tilde{\Psi}^{\prime c}=\tilde{\Psi}^{c} \underline{\tilde{N}}, \quad \underline{\tilde{N}}=\left(\begin{array}{cc}
\tilde{N} & 0 \\
0 & \tilde{N}
\end{array}\right), \quad \underline{D}=\underline{\tilde{N}} \underline{D}^{\prime} \underline{N} .
$$

Then we get

$$
\tilde{\Psi}^{c}\left(\underline{D} \Psi^{c}\right) L_{012}=\tilde{\Psi}^{c} \underline{\tilde{N}} \underline{D^{\prime}} \underline{N} \Psi^{c} L_{012}=\tilde{\Psi}^{\prime c}\left(\underline{D}^{\prime} \Psi^{\prime c}\right) L_{012}
$$


and we shall now study the form invariance of the mass term. All $s_{j}$ are determinants of a $\phi$ matrix, this implies

This gives

$$
\begin{aligned}
& s_{j}^{\prime}=\operatorname{det}\left(\phi^{\prime}\right)=\operatorname{det}(M \phi)=\operatorname{det}(M) \operatorname{det}(\phi)=r \mathrm{e}^{\mathrm{i} \theta} s_{j} \\
& s_{j}^{\prime *}=r \mathrm{e}^{-i \theta} s_{j}^{*}, \quad \rho_{2}^{\prime}=r \rho_{2} .
\end{aligned}
$$

$$
\begin{aligned}
& \chi^{\prime c}=\left(\begin{array}{cc}
\chi_{b}^{\prime} & \chi_{g}^{\prime} \\
\chi_{r}^{\prime} & 0
\end{array}\right) \\
& r^{2} \rho_{2}^{2} \chi^{\prime c}=\rho_{2}^{\prime 2} \chi^{\prime c}=\left(\begin{array}{cc}
\mathrm{re}^{-i \theta} M & 0 \\
0 & r \mathrm{e}^{i \theta} \hat{M}
\end{array}\right) \rho_{2}^{2} \chi^{c} \\
& \chi^{\prime c}=\left(\begin{array}{cc}
r^{-1} \mathrm{e}^{-i \theta} M & 0 \\
0 & r^{-1} \mathrm{e}^{i \theta} \hat{M}
\end{array}\right) \chi_{c}=\tilde{N}^{-1} \chi^{c} \\
& \tilde{\Psi}^{\prime c} \chi^{\prime c}=\tilde{\Psi^{c}} \tilde{N} \tilde{N}^{-1} \chi^{c}=\tilde{\Psi}^{c} \chi^{c} .
\end{aligned}
$$

Then the form invariance of the wave equation is equivalent to the condition on the mass term

$$
\begin{aligned}
& m_{2}^{\prime} \rho_{2}^{\prime}=m_{2} \rho_{2} \\
& m_{2}^{\prime} r=m_{2}
\end{aligned}
$$

linked to the existence of the Planck factor [13].

\subsection{Gauge Invariance of the Wave Equation}

Since we have previously proved the gauge invariance of the lepton part of the wave equation, it is reason enough to prove the gauge invariance of the quark part of the wave equation.

\subsubsection{Gauge Group Generated by $\underline{P}_{0}$}

We have here

$$
\begin{aligned}
& \underline{P}_{0}\left(\Psi^{c}\right)=\Psi^{c}\left(-\frac{1}{3} L_{21}\right) \\
& \Psi^{\prime c}=\left[\exp \left(\theta \underline{P}_{0}\right)\right]\left(\Psi^{c}\right)=\Psi^{c} \exp \left(-\frac{\theta}{3} L_{21}\right) \\
& B_{\mu}^{\prime}=B_{\mu}-\frac{2}{g_{1}} B_{\mu} .
\end{aligned}
$$

To get the gauge invariance of the wave equation we must get

$$
\chi^{\prime c}=\chi^{c} \exp \left(-\frac{\theta}{3} L_{21}\right), \quad \chi_{c}^{\prime}=\chi_{c} \exp \left(-\frac{\theta}{3} \gamma_{21}\right), \quad c=r, g, b .
$$

This is satisfied because

$$
\begin{aligned}
& \phi_{d c}^{\prime}=\phi_{d c} \mathrm{e}^{-i \frac{\theta}{3} \sigma_{3}}, \quad \phi_{u c}^{\prime}=\phi_{u c} \mathrm{e}^{-i \frac{\theta}{3} \sigma_{3}} \\
& \eta_{1 c}^{\prime *}=\mathrm{e}^{i \frac{\theta}{3}} \eta_{1 d c}^{*}, \quad \eta_{1 u c}^{\prime *}=\mathrm{e}^{i \frac{\theta}{3}} \eta_{1 u c}^{*}, \\
& \eta_{2 d c}^{\prime *}=\mathrm{e}^{i \frac{\theta}{3}} \eta_{2 d c}^{*}, \quad \eta_{2 u c}^{\prime *}=\mathrm{e}^{i \frac{\theta}{3}} \eta_{2 u c}^{*} . \\
& s_{j}^{\prime}=\mathrm{e}^{2 i \frac{\theta}{3}} s_{j}, \quad j=1,2, \cdots, 15 .
\end{aligned}
$$


All up terms in the matrix $\chi_{c}$ contain $s_{j}^{*} \phi_{d c} \sigma_{1}$ and $s_{j}^{*} \phi_{u c} \sigma_{1}$ terms. We get

$$
\begin{aligned}
& \phi_{d c}^{\prime}=\phi_{d c} \mathrm{e}^{-i \frac{\theta}{3} \sigma_{3}}=\mathrm{e}^{i \frac{\theta}{3}} \phi_{d c} \\
& s_{j}^{\prime *} \phi_{d c}^{\prime} \sigma_{1}=\mathrm{e}^{-i \frac{\theta}{3}} \phi_{d c} \sigma_{1}=\phi_{d c} \mathrm{e}^{\frac{\theta}{3} \sigma_{12}} \sigma_{1}=\phi_{d c} \sigma_{1} \mathrm{e}^{-\frac{\theta}{3} \sigma_{12}} \\
& \chi_{c}^{\prime}=\chi_{c} \exp \left(-\frac{\theta}{3} \gamma_{21}\right) \\
& \chi^{\prime c}=\chi^{c} \exp \left(-\frac{\theta}{3} L_{21}\right) .
\end{aligned}
$$

And we finally get

$$
\left(\underline{D}^{\prime} \Psi^{\prime c}\right) L_{012}+m_{2} \rho_{2}^{\prime} \chi^{\prime c}=\left[\left(\underline{D} \Psi^{c}\right) L_{012}+m_{2} \rho_{2} \chi^{c}\right] \exp \left(-\frac{\theta}{3} L_{21}\right)=0 .
$$

The wave equation with mass term is gauge invariant under the group generated by $\underline{P}_{0}$.

\subsubsection{Gauge Group Generated by $\underline{P}_{1}$}

We have here

$$
\begin{aligned}
& \underline{P}_{1}\left(\Psi^{c}\right)=\Psi^{c} L_{35} \\
& \Psi^{\prime c}=\left[\exp \left(\theta \underline{P}_{1}\right)\right]\left(\Psi^{c}\right)=\Psi^{c} \exp \left(\theta L_{35}\right) \\
& W_{\mu}^{\prime 1}=W_{\mu}^{1}-\frac{2}{g_{2}} \partial_{\mu} \theta .
\end{aligned}
$$

Since $\underline{P}_{1}\left(\Psi^{c}\right)=\Psi^{c} L_{35}$ we get

$$
\begin{aligned}
& \Psi^{\prime c}=\left[\exp \left(\theta \underline{P}_{1}\right)\right]\left(\Psi^{c}\right)=\Psi^{c} \exp \left(\theta L_{35}\right) \\
& \Psi_{c}^{\prime}=\Psi_{c} \mathrm{e}^{\theta_{\gamma / 3} i}, \quad c=r, g, b .
\end{aligned}
$$

We let

$$
C=\cos (\theta), \quad S=\sin (\theta) .
$$

Then (5.31) is equivalent to the system

or to the system

$$
\begin{aligned}
& \hat{\phi}_{d c}^{\prime}=C \hat{\phi}_{d c}-i S \hat{\phi}_{u c} \sigma_{3} \\
& \hat{\phi}_{u c}=C \hat{\phi}_{u c}-i S \hat{\phi}_{d c} \sigma_{3}
\end{aligned}
$$

We then get

$$
\begin{array}{ll}
\eta_{1 d c}^{\prime}=C \eta_{1 d c}-i S \eta_{1 u c}, & \eta_{1 d c}^{\prime *}=C \eta_{1 d c}^{*}+i S \eta_{1 u c}^{*} \\
\eta_{2 d c}^{\prime}=C \eta_{2 d c}-i S \eta_{2 u c}, & \eta_{2 d c}^{\prime *}=C \eta_{2 d c}^{*}+i S \eta_{2 u c}^{*} \\
\eta_{1 u c}^{\prime}=C \eta_{1 u c}-i S \eta_{1 d c}, & \eta_{1 u c}^{\prime *}=C \eta_{1 u c}^{*}+i S \eta_{1 d c}^{*} \\
\eta_{2 u c}^{\prime}=C \eta_{2 u c}-i S \eta_{2 d c}, & \eta_{2 u c}^{\prime *}=C \eta_{2 u c}^{*}+i S \eta_{2 d c}^{*} .
\end{array}
$$

$$
\begin{aligned}
& s_{1}^{\prime}=C^{2} s_{1}-S^{2} s_{4}+i C S\left(s_{10}-s_{14}\right) \\
& s_{4}^{\prime}=C^{2} s_{4}-S^{2} s_{1}+i C S\left(s_{10}-s_{14}\right) \\
& s_{10}^{\prime}=C^{2} s_{10}+S^{2} s_{14}+i C S\left(s_{1}+s_{4}\right)
\end{aligned}
$$




$$
s_{14}^{\prime}=C^{2} s_{14}+S^{2} s_{10}-i C S\left(s_{1}+s_{4}\right) .
$$

This implies

$$
s_{1}^{\prime} s_{1}^{\prime *}+s_{4}^{\prime} s_{4}^{\prime *}+s_{10}^{\prime} s_{10}^{\prime *}+s_{14}^{\prime} s_{14}^{\prime *}=s_{1} s_{1}^{*}+s_{4} s_{4}^{*}+s_{10} s_{10}^{*}+s_{14} s_{14}^{*} .
$$

Similarly, permuting colors, we get

$$
\begin{aligned}
& s_{2}^{\prime}=C^{2} s_{2}-S^{2} s_{5}+i C S\left(s_{11}-s_{15}\right) \\
& s_{5}^{\prime}=C^{2} s_{5}-S^{2} s_{2}+i C S\left(s_{11}-s_{15}\right) \\
& s_{11}^{\prime}=C^{2} s_{11}+S^{2} s_{15}+i C S\left(s_{2}+s_{5}\right) \\
& s_{15}^{\prime}=C^{2} s_{15}+S^{2} s_{11}-i C S\left(s_{2}+s_{5}\right) .
\end{aligned}
$$

This implies

$$
s_{2}^{\prime} s_{2}^{\prime *}+s_{5}^{\prime} s_{5}^{\prime *}+s_{11}^{\prime} s_{11}^{\prime *}+s_{15}^{\prime} s_{15}^{\prime *}=s_{2} s_{2}^{*}+s_{5} s_{5}^{*}+s_{11} s_{11}^{*}+s_{15} s_{15}^{*}
$$

and also

$$
\begin{aligned}
& s_{3}^{\prime}=C^{2} s_{3}-S^{2} s_{6}+i C S\left(s_{12}-s_{13}\right) \\
& s_{6}^{\prime}=C^{2} s_{6}-S^{2} s_{3}+i C S\left(s_{12}-s_{13}\right) \\
& s_{12}^{\prime}=C^{2} s_{12}+S^{2} s_{13}+i C S\left(s_{3}+s_{6}\right) \\
& s_{13}^{\prime}=C^{2} s_{13}+S^{2} s_{12}-i C S\left(s_{3}+s_{6}\right) .
\end{aligned}
$$

This implies

$$
s_{3}^{\prime} s_{3}^{\prime *}+s_{6}^{\prime} s_{6}^{\prime *}+s_{12}^{\prime} s_{12}^{\prime *}+s_{13}^{\prime} s_{13}^{\prime *}=s_{3} s_{3}^{*}+s_{6} s_{6}^{*}+s_{12} s_{12}^{*}+s_{13} s_{13}^{*} .
$$

\section{Moreover we get}

We then get

$$
s_{7}^{\prime}=s_{7}, \quad s_{8}^{\prime}=s_{8}, \quad s_{9}^{\prime}=s_{9} .
$$

$$
\rho^{\prime}=\rho
$$

Next we have

$$
\begin{aligned}
& \chi_{r}=\left(\begin{array}{ll}
A & B \\
\hat{B} & \hat{A}
\end{array}\right), \quad \chi_{r}^{\prime}=\left(\begin{array}{ll}
A^{\prime} & B^{\prime} \\
\hat{B}^{\prime} & \hat{A}^{\prime}
\end{array}\right) \\
& \hat{A}=\left(-s_{4} \hat{\phi}_{d g}+s_{6} \hat{\phi}_{d b}+s_{7} \hat{\phi}_{u r}+s_{12} \hat{\phi}_{u b}+s_{14} \hat{\phi}_{u g}\right) \sigma_{1} \\
& \hat{B}=\left(-s_{1} \hat{\phi}_{u g}+s_{3} \hat{\phi}_{u b}-s_{7} \hat{\phi}_{d r}-s_{10} \hat{\phi}_{d g}-s_{13} \hat{\phi}_{d b}\right) \sigma_{1}
\end{aligned}
$$

and we get

$$
\begin{aligned}
\hat{A}^{\prime} & =C \hat{A}-i S \hat{B} \sigma_{3} \\
\hat{B}^{\prime} & =C \hat{B}-i S \hat{A} \sigma_{3} \\
\chi_{r}^{\prime} & =\chi_{r}\left(\begin{array}{cc}
C & -i S \sigma_{3} \\
-i S \sigma_{3} & C
\end{array}\right)=\chi_{\mathrm{r}} \mathrm{e}^{\theta / 3 i} .
\end{aligned}
$$

Since we get the same relation for $g$ and $b$ colors we finally get

$$
\begin{aligned}
& \chi^{\prime c}=\chi^{c} \exp \left(\theta L_{35}\right), \\
& \left(\underline{D}^{\prime} \Psi^{\prime c}\right) L_{012}+m_{2} \rho_{2}^{\prime} \chi^{\prime c}=\left(\underline{D} \Psi^{c}\right) \exp \left(\theta L_{35}\right) L_{012}+m_{2} \rho_{2}^{\prime} \chi^{\prime c} \\
& =\left[\left(\underline{D}^{c}\right) L_{012}+m_{2} \rho_{2} \chi^{c}\right] \exp \left(\theta L_{35}\right)=0 .
\end{aligned}
$$


The wave equation with mass term is then gauge invariant under the group generated by $\underline{P}$.

5.2.3. Gauge Group Generated by $\underline{P}_{2}$ We have here

$$
\begin{aligned}
& \underline{P}_{2}\left(\Psi^{c}\right)=\Psi^{c} L_{0125} \\
& \Psi^{\prime c}=\left[\exp \left(\theta \underline{P}_{2}\right)\right]\left(\Psi^{c}\right)=\Psi^{c} \exp \left(\theta L_{0125}\right) \\
& W_{\mu}^{\prime 2}=W_{\mu}^{2}-\frac{2}{g_{2}} \partial_{\mu} \theta
\end{aligned}
$$

Since $\underline{P}_{2}\left(\Psi^{c}\right)=\Psi^{c} L_{0125}$ we get

$$
\begin{aligned}
& \Psi^{\prime c}=\left[\exp \left(\theta \underline{P}_{2}\right)\right]\left(\Psi^{c}\right)=\Psi^{c} \exp \left(\theta L_{0125}\right) \\
& \Psi_{c}^{\prime}=\Psi_{c} \mathrm{e}^{\theta / 3}, \quad c=r, g, b .
\end{aligned}
$$

We let

$$
C=\cos (\theta), \quad S=\sin (\theta) .
$$

Then (5.67) is equivalent to the system

$$
\begin{aligned}
& \hat{\phi}_{d c}^{\prime}=C \hat{\phi}_{d c}+S \hat{\phi}_{u c} \\
& \hat{\phi}_{u c}^{\prime}=C \hat{\phi}_{u c}-S \hat{\phi}_{d c}
\end{aligned}
$$

or to the system

$$
\begin{array}{cl}
\eta_{1 d c}^{\prime}=C \eta_{1 d c}+S \eta_{1 u c}, & \eta_{1 d c}^{\prime *}=C \eta_{1 d c}^{*}+S \eta_{1 u c}^{*} \\
\eta_{2 d c}^{\prime}=C \eta_{2 d c}+S \eta_{2 u c}, & \eta_{2 d c}^{\prime *}=C \eta_{2 d c}^{*}+S \eta_{2 u c}^{*} \\
\eta_{1 u c}^{\prime}=C \eta_{1 u c}-S \eta_{1 d c}, & \eta_{1 u c}^{\prime *}=C \eta_{1 u c}^{*}-S \eta_{1 d c}^{*} \\
\eta_{2 u c}^{\prime}=C \eta_{2 u c}-S \eta_{2 d c}, & \eta_{2 u c}^{\prime *}=C \eta_{2 u c}^{*}-S \eta_{2 d c}^{*}
\end{array}
$$

We then get

$$
\begin{aligned}
& s_{1}^{\prime}=C^{2} s_{1}+S^{2} s_{4}-C S s_{10}+C S s_{14} \\
& s_{4}^{\prime}=C^{2} s_{4}+S^{2} s_{1}+C S s_{10}-C S s_{14} \\
& s_{10}^{\prime}=C^{2} s_{10}+S^{2} s_{14}+C S s_{1}-C S s_{4} \\
& s_{14}^{\prime}=C^{2} s_{14}+S^{2} s_{10}-C S s_{1}+C S s_{4} .
\end{aligned}
$$

This implies

$$
s_{1}^{\prime} s_{1}^{\prime *}+s_{4}^{\prime} s_{4}^{\prime *}+s_{10}^{\prime} s_{10}^{\prime *}+s_{14}^{\prime} s_{14}^{\prime *}=s_{1} s_{1}^{*}+s_{4} s_{4}^{*}+s_{10} s_{10}^{*}+s_{14} s_{14}^{*} .
$$

Similarly, permuting colors, we get

$$
\begin{gathered}
s_{2}^{\prime}=C^{2} s_{2}+S^{2} s_{5}-C S s_{11}+C S s_{15} \\
s_{5}^{\prime}=C^{2} s_{5}+S^{2} s_{2}+C S s_{11}-C S s_{15} \\
s_{11}^{\prime}=C^{2} s_{11}+S^{2} s_{15}+C S s_{2}-C S s_{5} \\
s_{15}^{\prime}=C^{2} s_{15}+S^{2} s_{11}-C S s_{2}+C S s_{5} .
\end{gathered}
$$

This implies 


$$
s_{2}^{\prime} s_{2}^{\prime *}+s_{5}^{\prime} s_{5}^{\prime *}+s_{11}^{\prime} s_{11}^{\prime *}+s_{15}^{\prime} s_{15}^{\prime *}=s_{2} s_{2}^{*}+s_{5} s_{5}^{*}+s_{11} s_{11}^{*}+s_{15} s_{15}^{*}
$$

and also

$$
\begin{aligned}
& s_{3}^{\prime}=C^{2} s_{3}+S^{2} s_{6}-C S s_{12}+C S s_{13} \\
& s_{6}^{\prime}=C^{2} s_{6}+S^{2} s_{3}+C S s_{12}-C S s_{13} \\
& s_{12}^{\prime}=C^{2} s_{12}+S^{2} s_{13}+C S s_{3}-C S s_{6} \\
& s_{13}^{\prime}=C^{2} s_{13}+S^{2} s_{12}-C S s_{3}+C S s_{6} .
\end{aligned}
$$

This implies

$$
s_{3}^{\prime} s_{3}^{\prime *}+s_{6}^{\prime} s_{6}^{\prime *}+s_{12}^{\prime} s_{12}^{\prime *}+s_{13}^{\prime} s_{13}^{\prime *}=s_{3} s_{3}^{*}+s_{6} s_{6}^{*}+s_{12} s_{12}^{*}+s_{13} s_{13}^{*} .
$$

Moreover we get

We then get

$$
s_{7}^{\prime}=s_{7}, \quad s_{8}^{\prime}=s_{8}, \quad s_{9}^{\prime}=s_{9} .
$$

$$
\rho^{\prime}=\rho
$$

Next we get with (5.56)

$$
\begin{aligned}
\hat{A}^{\prime} & =C \hat{A}-S \hat{B} \sigma_{3} \\
\hat{B}^{\prime} & =C \hat{B}+S \hat{A} \sigma_{3} \\
\chi_{r}^{\prime} & =\chi_{r}\left(\begin{array}{cc}
C & -S \sigma_{3} \\
S \sigma_{3} & C
\end{array}\right)=\chi_{r} \mathrm{e}^{-\theta \gamma_{3}} .
\end{aligned}
$$

Since we get the same relation for $g$ and $b$ colors we finally get

$$
\begin{aligned}
& \chi^{\prime c}=\chi^{c} \exp \left(-\theta L_{0125}\right), \\
& \left(\underline{D}^{\prime} \Psi^{\prime c}\right) L_{012}+m_{2} \rho_{2}^{\prime} \chi^{\prime c}=\left(\underline{D} \Psi^{c}\right) \exp \left(\theta L_{0125}\right) L_{012}+m_{2} \rho_{2}^{\prime} \chi^{\prime c} \\
& =\left[\left(\underline{D}^{c}\right) L_{012}+m_{2} \rho_{2} \chi^{c}\right] \exp \left(-\theta L_{0125}\right)=0 .
\end{aligned}
$$

The wave equation with mass term is then gauge invariant under the group generated by $\underline{P}_{2}$.

\subsubsection{Gauge Group Generated by $\underline{P}_{3}$}

We have here

$$
\begin{aligned}
& \underline{P}_{3}\left(\Psi^{c}\right)=\Psi^{c} L_{3012} \\
& \Psi^{\prime c}=\left[\exp \left(\theta \underline{P}_{3}\right)\right]\left(\Psi^{c}\right)=\Psi^{c} \exp \left(\theta L_{3012}\right) \\
& W_{\mu}^{\prime 3}=W_{\mu}^{3}-\frac{2}{g_{2}} \partial_{\mu} \theta .
\end{aligned}
$$

Since $\underline{P}_{3}\left(\Psi^{c}\right)=\Psi^{c} L_{3012}$ we get

$$
\begin{aligned}
& \Psi^{\prime c}=\left[\exp \left(\theta \underline{P}_{3}\right)\right]\left(\Psi^{c}\right)=\Psi^{c} \exp \left(\theta L_{3012}\right) \\
& \Psi_{c}^{\prime}=\Psi_{c} \mathrm{e}^{\theta / 3012}, \quad c=r, g, b .
\end{aligned}
$$

Then (5.97) is equivalent to the system

$$
\begin{aligned}
& \hat{\phi}_{d c}^{\prime}=\mathrm{e}^{\mathrm{i} \theta} \hat{\phi}_{d c} \\
& \hat{\phi}_{u c}^{\prime}=\mathrm{e}^{-i \theta} \hat{\phi}_{u c}
\end{aligned}
$$

or to the system 


$$
\begin{array}{ll}
\eta_{1 d c}^{\prime}=\mathrm{e}^{i \theta} \eta_{1 d c}, & \eta_{1 d c}^{\prime *}=\mathrm{e}^{-i \theta} \eta_{1 d c}^{*} \\
\eta_{2 d c}^{\prime}=\mathrm{e}^{i \theta} \eta_{2 d c}, & \eta_{2 d c}^{\prime *}=\mathrm{e}^{-i \theta} \eta_{2 d c}^{*} \\
\eta_{1 u c}^{\prime}=\mathrm{e}^{-i \theta} \eta_{1 u c}, & \eta_{1 u c}^{\prime *}=\mathrm{e}^{\mathrm{i} \theta} \eta_{1 u c}^{*} \\
\eta_{2 u c}^{\prime}=\mathrm{e}^{-i \theta} \eta_{2 u c}, & \eta_{2 u c}^{\prime *}=\mathrm{e}^{i \theta} \eta_{2 u c}^{*} .
\end{array}
$$

We then get

$$
\begin{aligned}
& s_{1}^{\prime}=\mathrm{e}^{2 i \theta} s_{1}, \quad s_{2}^{\prime}=\mathrm{e}^{2 i \theta} s_{2}, \quad s_{3}^{\prime}=\mathrm{e}^{2 i \theta} s_{3} \\
& s_{4}^{\prime}=\mathrm{e}^{-2 i \theta} s_{4}, \quad s_{5}^{\prime}=\mathrm{e}^{-2 i \theta} s_{5}, \quad s_{6}^{\prime}=\mathrm{e}^{-2 i \theta} s_{6} \\
& s_{7}^{\prime}=s_{7}, \quad s_{8}^{\prime}=s_{8}, \quad s_{9}^{\prime}=s_{9} \\
& s_{10}^{\prime}=s_{10}, \quad s_{11}^{\prime}=s_{11}, \quad s_{12}^{\prime}=s_{12} \\
& s_{13}^{\prime}=s_{13}, \quad s_{14}^{\prime}=s_{14}, \quad s_{15}^{\prime}=s_{15} .
\end{aligned}
$$

This implies

$$
\rho^{\prime}=\rho .
$$

Next we get with (5.56)

$$
\begin{aligned}
\hat{A}^{\prime} & =\mathrm{e}^{-i \theta} \hat{A}, \quad A^{\prime}=\mathrm{e}^{i \theta} A \\
\hat{B}^{\prime} & =\mathrm{e}^{i \theta} \hat{B}, \quad B^{\prime}=\mathrm{e}^{-i \theta} B \\
\chi_{r}^{\prime} & =\chi_{r}\left(\begin{array}{cc}
\mathrm{e}^{\mathrm{i} \theta} & 0 \\
0 & \mathrm{e}^{-i \theta}
\end{array}\right)=\chi_{r} \mathrm{e}^{\theta \mathrm{i}} .
\end{aligned}
$$

Since we get the same relation for $g$ and $b$ colors we finally get

$$
\begin{aligned}
& \chi^{\prime c}=\chi^{c} \exp \left(-\theta L_{3012}\right), \\
& \left(\underline{D}^{\prime} \Psi^{\prime c}\right) L_{012}+m_{2} \rho_{2}^{\prime} \chi^{\prime c}=\left(\underline{D} \Psi^{c}\right) \exp \left(\theta L_{3012}\right) L_{012}+m_{2} \rho_{2}^{\prime} \chi^{\prime c} \\
& =\left[\left(\underline{D}^{c}\right) L_{012}+m_{2} \rho_{2} \chi^{c}\right] \exp \left(-\theta L_{3012}\right)=0 .
\end{aligned}
$$

The wave equation with mass term is then gauge invariant under the group generated by $\underline{P}_{3}$.

\subsubsection{Gauge Group Generated by $\Gamma_{1}$}

We use now the gauge transformation

$$
\begin{aligned}
& \Psi_{r}^{\prime}=C \Psi_{r}+S \mathbf{i} \Psi_{g}, \quad C=\cos (\theta), \quad S=\sin (\theta) \\
& \Psi_{g}^{\prime}=C \Psi_{g}+S \mathbf{i} \Psi_{r} \\
& \Psi_{b}^{\prime}=\Psi_{b} .
\end{aligned}
$$

We can then forget here $\Psi_{b}$. The gauge invariance signifies that the system

$$
\begin{aligned}
& \partial \Psi_{r}=-\frac{g_{3}}{2} \mathbf{G}^{1} \mathbf{i} \Psi_{g}+m_{2} \rho_{2} \chi_{r} \gamma_{012}, \\
& \partial \Psi_{g}=-\frac{g_{3}}{2} \mathbf{G}^{1} \mathbf{i} \Psi_{r}+m_{2} \rho_{2} \chi_{g} \gamma_{012}
\end{aligned}
$$

must be equivalent to the system

$$
\begin{aligned}
& \partial \Psi_{r}^{\prime}=-\frac{g_{3}}{2} \mathbf{G}^{\prime} \mathbf{i} \Psi_{g}^{\prime}+m_{2} \rho_{2}^{\prime} \chi_{r}^{\prime} \gamma_{012}, \\
& \partial \Psi_{g}^{\prime}=-\frac{g_{3}}{2} \mathbf{G}^{\prime} \mathbf{i} \Psi_{r}^{\prime}+m_{2} \rho_{2}^{\prime} \chi_{g}^{\prime} \gamma_{012} .
\end{aligned}
$$


Using relations (5.117) and (5.118) the system (5.121) is equivalent to (5.120) if and only if

$$
\mathbf{G}^{\prime 1}=\mathbf{G}^{1}-\frac{2}{g_{3}} \partial \theta
$$

We name $f_{1}$ the gauge transformation

$$
f_{1}: \Psi^{c} \mapsto \underline{\mathbf{i}} \Gamma_{1}\left(\Psi^{c}\right)=\left(\begin{array}{cc}
0 & \mathbf{i} \Psi_{g} \\
\mathbf{i} \Psi_{r} & 0
\end{array}\right)
$$

which implies with $C=\cos (\theta)$ and $S=\sin (\theta)$

$$
\begin{aligned}
{\left[\exp \left(\theta f_{1}\right)\right]\left(\Psi^{c}\right)=} & \left(\begin{array}{cc}
0 & C \Psi_{r}+S \mathbf{i} \Psi_{g} \\
C \Psi_{g}+S \mathbf{i} \Psi_{r} & \Psi_{b}
\end{array}\right)=\left(\begin{array}{cc}
0 & \Psi_{r}^{\prime} \\
\Psi_{g}^{\prime} & \Psi_{b}^{\prime}
\end{array}\right) \\
& \Psi_{r}^{\prime}=C \Psi_{r}+S \mathbf{i} \Psi_{g} \\
& \Psi_{g}^{\prime}=C \Psi_{g}+S \mathbf{i} \Psi_{r} \\
& \Psi_{b}^{\prime}=\Psi_{b} .
\end{aligned}
$$

The equality (5.117) is equivalent to the system

$$
\begin{array}{cc}
\eta_{1 d r}^{\prime *}=C \eta_{1 d r}^{*}+i S \eta_{1 d g}^{*}, & \eta_{1 u r}^{\prime *}=C \eta_{1 u r}^{*}+i S \eta_{1 u g}^{*} \\
\eta_{2 d r}^{\prime *}=C \eta_{2 d r}^{*}+i S \eta_{2 d g}^{*}, & \eta_{2 u r}^{\prime *}=C \eta_{2 u r}^{*}+i S \eta_{2 u g}^{*} .
\end{array}
$$

The equality (5.118) is equivalent to the system

$$
\begin{array}{ll}
\eta_{1 d g}^{\prime *}=C \eta_{1 d g}^{*}+i S \eta_{1 d r}^{*}, & \eta_{1 u g}^{\prime *}=C \eta_{1 u g}^{*}+i S \eta_{1 u r}^{*} \\
\eta_{2 d g}^{\prime *}=C \eta_{2 d g}^{*}+i S \eta_{2 d r}^{*}, & \eta_{2 u g}^{\prime *}=C \eta_{2 u g}^{*}+i S \eta_{2 u r}^{*} .
\end{array}
$$

This gives for the invariant scalars $s_{j}$

$$
\begin{aligned}
& s_{1}^{\prime}=s_{1}, \quad s_{4}^{\prime}=s_{4}, \quad s_{9}^{\prime}=s_{9} \\
& s_{2}^{\prime}=C s_{2}-i S s_{3}, \quad s_{3}^{\prime}=C s_{3}-i S s_{2} \\
& s_{5}^{\prime}=C s_{5}-i S s_{6}, \quad s_{6}^{\prime}=C s_{6}-i S s_{5} \\
& s_{11}^{\prime}=C s_{11}+i S s_{13}, \quad s_{13}^{\prime}=C s_{13}+i S s_{11} \\
& s_{12}^{\prime}=C s_{12}+i S s_{15}, \quad s_{15}^{\prime}=C s_{15}+i S s_{12} \\
& s_{7}^{\prime}=C^{2} s_{7}-S^{2} s_{8}+i C s_{10}+i C S s_{14} \\
& s_{8}^{\prime}=C^{2} s_{8}-S^{2} s_{7}+i C S s_{14}+i C S s_{10} \\
& s_{10}^{\prime}=C^{2} s_{10}-S^{2} s_{14}+i C S s_{7}+i C S s_{8} \\
& s_{14}^{\prime}=C^{2} s_{14}-S^{2} s_{10}+i C S s_{8}+i C S s_{7} .
\end{aligned}
$$

We then get

$$
\begin{aligned}
& s_{2}^{\prime} s_{2}^{\prime *}+s_{3}^{\prime} s_{3}^{\prime *}=s_{2} s_{2}^{*}+s_{3} s_{3}^{*} \\
& s_{5}^{\prime} s_{5}^{\prime *}+s_{6}^{\prime} s_{6}^{\prime *}=s_{5} s_{5}^{*}+s_{6} s_{6}^{*} \\
& s_{11}^{\prime} s_{11}^{\prime *}+s_{13}^{\prime} s_{13}^{\prime *}=s_{11} s_{11}^{*}+s_{13} s_{13}^{*} \\
& s_{12}^{\prime} s_{12}^{\prime *}+s_{15}^{\prime} s_{15}^{\prime *}=s_{12} s_{12}^{*}+s_{15} s_{15}^{*} \\
& s_{7}^{\prime} s_{7}^{\prime *}+s_{8}^{\prime} s_{8}^{\prime *}+s_{10}^{\prime} s_{10}^{\prime *}+s_{14}^{\prime} s_{14}^{\prime *}=s_{7} s_{7}^{*}+s_{8} s_{8}^{*}+s_{10} s_{10}^{*}+s_{14} s_{14}^{*}
\end{aligned}
$$


Next we let

$$
\rho^{\prime}=\rho .
$$

$$
\begin{array}{ll}
\chi_{r}=\left(\begin{array}{ll}
A_{r} & B_{r} \\
\hat{B}_{r} & \hat{A}_{r}
\end{array}\right), \quad \chi_{r}^{\prime}=\left(\begin{array}{ll}
A_{r}^{\prime} & B_{r}^{\prime} \\
\hat{B}_{r}^{\prime} & \hat{A}_{r}^{\prime}
\end{array}\right) \\
\chi_{g}=\left(\begin{array}{ll}
A_{g} & B_{g} \\
\hat{B}_{g} & \hat{A}_{g}
\end{array}\right), \quad \chi_{g}^{\prime}=\left(\begin{array}{ll}
A_{g}^{\prime} & B_{g}^{\prime} \\
\hat{B}_{g}^{\prime} & \hat{A}_{g}^{\prime}
\end{array}\right)
\end{array}
$$

and we get with (B.17) and (B.18)

$$
\begin{array}{ll}
A_{r}^{\prime}=C A_{r}-i S A_{g}, & B_{r}^{\prime}=C B_{r}-i S B_{g} \\
A_{g}^{\prime}=C A_{g}-i S A_{r}, & B_{g}^{\prime}=C B_{g}-i S B_{r} .
\end{array}
$$

This gives the awaited result

$$
\begin{aligned}
& \rho^{\prime}=\rho \\
& \chi_{r}^{\prime}=C \chi_{r}-S \mathbf{i} \chi_{g} \\
& \chi_{g}^{\prime}=C \chi_{g}-S \mathbf{i} \chi_{r} .
\end{aligned}
$$

The change of sign of the phase between (5.117) and (5.152) comes from the anticommutation between $\mathbf{i}$ and $\partial$.

\subsubsection{Gauge Groups Generated by $\Gamma_{k}, k>1$}

We use with $k=2$ the gauge transformation

$$
\begin{aligned}
\Psi_{r}^{\prime} & =C \Psi_{r}+S \Psi_{g}, \quad C=\cos (\theta), \quad S=\sin (\theta) \\
\Psi_{g}^{\prime} & =C \Psi_{g}-S \Psi_{r} \\
\Psi_{b}^{\prime} & =\Psi_{b} .
\end{aligned}
$$

The gauge invariance signifies that the system

$$
\begin{aligned}
& \partial \Psi_{r}=-\frac{g_{3}}{2} \mathbf{G}^{2} \Psi_{g}+m_{2} \rho_{2} \chi_{r} \gamma_{012}, \\
& \partial \Psi_{g}=\frac{g_{3}}{2} \mathbf{G}^{2} \Psi_{r}+m_{2} \rho_{2} \chi_{g} \gamma_{012}
\end{aligned}
$$

must be equivalent to the system

$$
\begin{aligned}
& \partial \Psi_{r}^{\prime}=-\frac{g_{3}}{2} \mathbf{G}^{\prime 2} \Psi_{g}^{\prime}+m_{2} \rho_{2}^{\prime} \chi_{r}^{\prime} \gamma_{012}, \\
& \partial \Psi_{g}^{\prime}=\frac{g_{3}}{2} \mathbf{G}^{\prime 2} \Psi_{r}^{\prime}+m_{2} \rho_{2}^{\prime} \chi_{g}^{\prime} \gamma_{012} .
\end{aligned}
$$

Using relations (5.154) and (5.155) the system (5.158) is equivalent to (5.157) if and only if

$$
\mathbf{G}^{\prime 2}=\mathbf{G}^{2}-\frac{2}{g_{3}} \partial \theta
$$

because we get

$$
\begin{aligned}
& \rho^{\prime}=\rho \\
& \chi_{r}^{\prime}=C \chi_{r}+S \chi_{g} \\
& \chi_{g}^{\prime}=C \chi_{g}-S \chi_{r} .
\end{aligned}
$$

The case $k=3$ is detailed in C. 1 and the case $k=8$ is detailed in C.2. Cases $k=4$ and $k=6$ are 
similar to $k=1$ and cases $k=5$ and $k=7$ are similar to $k=2$ by permutation of indexes of color.

\section{Concluding Remarks}

From experimental results obtained in the accelerators physicists have built what is now known as the "standard model". This model is generally thought to be a part of quantum field theory, itself a part of axiomatic quantum mechanics. One of these axioms is that each state describing a physical situation follows a Schrödinger wave equation. Since this wave equation is not relativistic and does not account for the spin $1 / 2$ which is necessary to any fermion, the standard model has evidently not followed the axiom and has used instead a Dirac equation to describe fermions. Our work also starts with the Dirac equation. This wave equation is the linear approximation of our nonlinear homogeneous equation of the electron.

The wave equation presented here is a wave equation for a classical wave, a function of space and time with value into a Clifford algebra. It is not a quantized wave with value into a Hilbertian space of operators. Nevertheless and consequently we get most of the aspects of the standard model, for instance the fact that leptons are insensitive to strong interactions. The standard model is much stronger than generally thought. For instance we firstly did not use the link between the wave of the particle and the wave of the antiparticle, but then we needed a greater Clifford algebra and we could not get the necessary link between reversions ${ }^{1}$ that we used in our wave equation. We also needed the existence of the inverse to build the wave of a system of particles from the waves of its components. And we got two general identities which existed only if all parts of the general wave were left waves, only the electron having also a right wave.

The most important property of the general wave is its form invariance under a group including the covering group of the restricted Lorentz group. Our group does not explain why space and time are oriented, but it respects these orientations. The physical time is then compatible with thermodynamics, and the physical space is compatible with the violation of parity by weak interactions.

The wave accounts for all particles and anti-particles of the first generation. We have also given [2] [8] [9] [13] the reason of the existence of three generations; it is simply the dimension of our physical space. Since the $S U$ (3) gauge group of chromodynamics acts independently from the index of generations, the physical quarks may be combinations of waves of different generations. Quarks composing protons and neutrons are such combinations. Our wave equation allows only two masses at each generation, one for the lepton part of the wave, the other one for the two quarks. The mixing can give a different mass for the two quarks of each generation.

Since the wave equation with mass term is gauge invariant, there is no necessity to use the mechanism of spontaneous symmetry breaking. The scalar boson certainly exists, but it does not explain the masses.

A wave equation is only a beginning. It shall be necessary to study also the boson part of the standard model and the systems of fermions, from this wave equation. A construction of the wave of a system of identical particles is possible and compatible with the Pauli principle [1] [7].

\section{References}

[1] Daviau, C. and Bertrand, J. (2014) New Insights in the Standard Model of Quantum Physics in Clifford Algebra. JePublie, Pouillé-les-Coteaux. http://hal.archives-ouvertes.fr/hal-00907848

[2] Daviau, C. and Bertrand, J. (2014) Journal of Modern Physics, 5, 1001-1022. http://dx.doi.org/10.4236/jmp.2014.511102

[3] Daviau, C. (1993) Equation de Dirac non linéaire. Ph.D. Thesis, Université de Nantes, Nantes.

[4] Daviau, C. (1997) Advances in Applied Clifford Algebras, 7, 175-194.

[5] Daviau, C. (2005) Annales de la Fondation Louis de Broglie, 30, 409-428.

[6] Daviau, C. (2011) L’espace-temps double. JePublie, Pouillé-les-coteaux.

[7] Daviau, C. (2012) Advances in Applied Clifford Algebras, 22, 611-623. http://dx.doi.org/10.1007/s00006-012-0351-7

[8] Daviau, C. (2012) Double Space-Time and More. JePublie, Pouillé-les-Coteaux.

[9] Daviau, C. (2012) Nonlinear Dirac Equation, Magnetic Monopoles and Double Space-Time. CISP, Cambridge.

[10] Deheuvels, R. (1993) Tenseurs et spineurs. PUF, Paris.

${ }^{1}$ The reversion is an anti-isomorphism changing the order of any product (see [1] 1.1). It is specific to each Clifford algebra. The Appendix A explains the link between the reversion in $C l_{1,3}$ and the reversion in $C l_{1,5}$. 
[11] Hestenes, D. (1986) A Unified Language for Mathematics and Physics and Clifford Algebra and the Interpretation of Quantum Mechanics. In: Chisholm, J.S.R. and Common, A.K., Eds., Clifford Algebras and Their Applications in Mathematics and Physics, Reidel, Dordrecht, 1-23.

[12] Weinberg, S. (1967) Physical Review Letters, 19, 1264-1266.

http://dx.doi.org/10.1103/PhysRevLett.19.1264

[13] Daviau, C. (2014) Gauge Group of the Standard Model in $\mathrm{Cl}_{1,5}$. ICCA10, Tartu. http://hal.archives-ouvertes.fr/hal-01055145

[14] de Broglie, L. (1924) Annales de la Fondation Louis de Broglie, 17. 


\section{Appendix A. Calculation of the Reverse in $\mathrm{Cl}_{1,5}$}

Here indexes $\mu, v, \rho, \ldots$ have value $0,1,2,3$ and indexes $a, b, c, d, e$ have value $0,1,2,3,4,5$. We use ${ }^{2}$ the following matrix representation of $\mathrm{Cl}_{1,5}$ :

$$
\begin{aligned}
& L_{\mu}=\left(\begin{array}{cc}
0 & \gamma_{\mu} \\
\gamma_{\mu} & 0
\end{array}\right) ; \quad L_{4}=\left(\begin{array}{cc}
0 & -I_{4} \\
I_{4} & 0
\end{array}\right) ; \quad L_{5}=\left(\begin{array}{cc}
0 & \mathbf{i} \\
\mathbf{i} & 0
\end{array}\right) ; \quad \mathbf{i}=\left(\begin{array}{cc}
i I_{2} & 0 \\
0 & -i I_{2}
\end{array}\right) ; \\
& \gamma_{0}=\gamma^{0}=\left(\begin{array}{cc}
0 & I_{2} \\
I_{2} & 0
\end{array}\right) ; \quad \gamma_{j}=-\gamma^{j}=\left(\begin{array}{cc}
0 & \sigma_{j} \\
-\sigma_{j} & 0
\end{array}\right) ; \quad j=1,2,3 .
\end{aligned}
$$

where $\sigma_{j}$ are Pauli matrices. This gives

$$
\begin{aligned}
& L_{\mu v}=L_{\mu} L_{v}=\left(\begin{array}{cc}
0 & \gamma_{\mu} \\
\gamma_{\mu} & 0
\end{array}\right)\left(\begin{array}{cc}
0 & \gamma_{\nu} \\
\gamma_{\nu} & 0
\end{array}\right)=\left(\begin{array}{cc}
\gamma_{\mu v} & 0 \\
0 & \gamma_{\mu v}
\end{array}\right) \\
& L_{\mu v \rho}=L_{\mu v} L_{\rho}=\left(\begin{array}{cc}
\gamma_{\mu v} & 0 \\
0 & \gamma_{\mu v}
\end{array}\right)\left(\begin{array}{cc}
0 & \gamma_{\rho} \\
\gamma_{\rho} & 0
\end{array}\right)=\left(\begin{array}{cc}
0 & \gamma_{\mu v \rho} \\
\gamma_{\mu v \rho} & 0
\end{array}\right) \\
& L_{0123}=L_{01} L_{23}=\left(\begin{array}{cc}
\gamma_{0123} & 0 \\
0 & \gamma_{0123}
\end{array}\right)=\left(\begin{array}{ll}
\mathbf{i} & 0 \\
0 & \mathbf{i}
\end{array}\right) .
\end{aligned}
$$

We get also

$$
\begin{aligned}
& L_{45}=L_{4} L_{5}=\left(\begin{array}{cc}
0 & -I_{4} \\
I_{4} & 0
\end{array}\right)\left(\begin{array}{ll}
0 & \mathbf{i} \\
\mathbf{i} & 0
\end{array}\right)=\left(\begin{array}{cc}
-\mathbf{i} & 0 \\
0 & \mathbf{i}
\end{array}\right)=-L_{54} \\
& L_{012345}=\left(\begin{array}{ll}
\mathbf{i} & 0 \\
0 & \mathbf{i}
\end{array}\right)\left(\begin{array}{ll}
-\mathbf{i} & 0 \\
0 & \mathbf{i}
\end{array}\right)=\left(\begin{array}{cc}
I_{4} & 0 \\
0 & -I_{4}
\end{array}\right) \\
& L_{01235}=L_{0123} L_{5}=\left(\begin{array}{ll}
\mathbf{i} & 0 \\
0 & \mathbf{i}
\end{array}\right)\left(\begin{array}{ll}
0 & \mathbf{i} \\
\mathbf{i} & 0
\end{array}\right)=\left(\begin{array}{cc}
0 & -I_{4} \\
-I_{4} & 0
\end{array}\right) .
\end{aligned}
$$

Similarly we get ${ }^{3}$

$$
\begin{aligned}
& L_{\mu 4}=\left(\begin{array}{cc}
\gamma_{\mu} & 0 \\
0 & -\gamma_{\mu}
\end{array}\right), \quad L_{\mu 5}=\left(\begin{array}{cc}
\gamma_{\mu} \mathbf{i} & 0 \\
0 & \gamma_{\mu} \mathbf{i}
\end{array}\right) \\
& L_{\mu v 4}=\left(\begin{array}{cc}
0 & -\gamma_{\mu v} \\
\gamma_{\mu v} & 0
\end{array}\right), \quad L_{\mu v 5}=\left(\begin{array}{cc}
0 & \gamma_{\mu v} \mathbf{i} \\
\gamma_{\mu v} \mathbf{i} & 0
\end{array}\right) \\
& L_{\mu v \rho 4}=\left(\begin{array}{cc}
\gamma_{\mu \nu \rho} & 0 \\
0 & -\gamma_{\mu \nu \rho}
\end{array}\right), \quad L_{\mu v \rho 5}=\left(\begin{array}{cc}
\gamma_{\mu v \rho} \mathbf{i} & 0 \\
0 & \gamma_{\mu \nu \rho} \mathbf{i}
\end{array}\right) \\
& L_{\mu 45}=\left(\begin{array}{cc}
0 & \gamma_{\mu} \mathbf{i} \\
-\gamma_{\mu} \mathbf{i} & 0
\end{array}\right), \quad L_{\mu v 45}=\left(\begin{array}{cc}
-\gamma_{\mu v} \mathbf{i} & 0 \\
0 & \gamma_{\mu v} \mathbf{i}
\end{array}\right) \\
& L_{\mu v \rho 45}=\left(\begin{array}{cc}
0 & \gamma_{\mu \nu \rho} \mathbf{i} \\
-\gamma_{\mu v \rho} \mathbf{i} & 0
\end{array}\right), \quad L_{01234}=\left(\begin{array}{cc}
0 & -\mathbf{i} \\
\mathbf{i} & 0
\end{array}\right) .
\end{aligned}
$$

Scalar and pseudo-scalar terms read

\footnotetext{
${ }^{2} I_{2}, \quad I_{4}, \quad I_{8}$ are unit matrices. The identification process allowing to include $\mathbb{R}$ in each real Clifford algebra allows to read $a$ instead of $a I_{n}$ for any complex number $a$.

${ }^{3} \mathbf{i}$ anti-commutes with any odd element in space-time algebra and commutes with any even element.
} 


$$
\begin{gathered}
\alpha I_{8}+\omega L_{012345}=\left(\begin{array}{cc}
(\alpha+\omega) I_{4} & 0 \\
0 & (\alpha-\omega) I_{4}
\end{array}\right) \\
\alpha I_{8}-\omega L_{012345}=\left(\begin{array}{cc}
(\alpha-\omega) I_{4} & 0 \\
0 & (\alpha+\omega) I_{4}
\end{array}\right) .
\end{gathered}
$$

For the calculation of the 1-vector term

$$
N^{a} L_{a}=N^{4} L_{4}+N^{5} L_{5}+N^{\mu} L_{\mu}
$$

we let

$$
\beta=N^{4}, \quad \delta=N^{5}, \quad \mathbf{a}=N^{\mu} \gamma_{\mu} .
$$

This gives

$$
N^{a} L_{a}=\left(\begin{array}{cc}
0 & -\beta I_{4}+\delta \mathbf{i}+\mathbf{a} \\
\beta I_{4}+\delta \mathbf{i}+\mathbf{a} & 0
\end{array}\right) .
$$

For the calculation of the 2-vector term

$$
N^{a b} L_{a b}=N^{45} L_{45}+N^{\mu 4} L_{\mu 4}+N^{\mu 5} L_{\mu 5}+N^{\mu v} L_{\mu v}
$$

we let

$$
\epsilon=N^{45}, \quad \mathbf{b}=N^{\mu 4} \gamma_{\mu}, \quad \mathbf{c}=N^{\mu 5} \gamma_{\mu}, \quad \mathbf{A}=N^{\mu v} \gamma_{\mu v} .
$$

This gives

$$
N^{a b} L_{a b}=\left(\begin{array}{cc}
-\epsilon \mathbf{i}+\mathbf{b}-\mathbf{i c}+\mathbf{A} & 0 \\
0 & \epsilon \mathbf{i}-\mathbf{b}-\mathbf{i c}+\mathbf{A}
\end{array}\right) .
$$

For the calculation of the 3-vector term

$$
N^{a b c} L_{a b c}=N^{\mu 45} L_{\mu 45}+N^{\mu v 4} L_{\mu v 4}+N^{\mu \nu 5} L_{\mu \nu 5}+N^{\mu v \rho} L_{\mu \nu \rho}
$$

we let

$$
\mathbf{d}=N^{\mu 45} \gamma_{\mu}, \quad \mathbf{B}=N^{\mu \nu 4} \gamma_{\mu \nu}, \quad \mathbf{C}=N^{\mu \nu 5} \gamma_{\mu \nu}, \quad \mathbf{i e}=N^{\mu \nu \rho} \gamma_{\mu \nu \rho} .
$$

This gives with (A.3) and (A.9)

$$
N^{a b c} L_{a b c}=\left(\begin{array}{cc}
0 & \mathbf{d i}-\mathbf{B}+\mathbf{i C}+\mathbf{i e} \\
\mathbf{i d}+\mathbf{B}+\mathbf{i C}+\mathbf{i e} & 0
\end{array}\right) .
$$

For the calculation of the 4-vector term

$$
N^{a b c d} L_{a b c d}=N^{\mu \nu 45} L_{\mu v 45}+N^{\mu \nu \rho 4} L_{\mu \nu \rho 4}+N^{\mu \nu \rho 5} L_{\mu \nu \rho 5}+N^{0123} L_{0123}
$$

we let

$$
\mathbf{D}=N^{\mu \nu 45} \gamma_{\mu \nu}, \quad \text { if }=N^{\mu \nu \rho 4} \gamma_{\mu \nu \rho}, \quad \text { ig }=N^{\mu v \rho 5} \gamma_{\mu \nu \rho}, \quad \zeta=N^{0123} .
$$

This gives with (A.4) and (A.10)

$$
N^{a b c d} L_{a b c d}=\left(\begin{array}{cc}
-\mathbf{i D}+\mathbf{i f}+\mathbf{g}+\zeta \mathbf{i} & 0 \\
0 & \mathbf{i D}-\mathbf{i f}+\mathbf{g}+\zeta \mathbf{i}
\end{array}\right) .
$$

For the calculation of the pseudo-vector term

$$
N^{\text {abcde }} L_{\text {abcde }}=N^{\mu v \rho 45} L_{\mu v \rho 45}+N^{01234} L_{01234}+N^{01235} L_{01235}
$$

we let 


$$
\text { ih }=N^{\mu v \rho 45} \gamma_{\mu \nu \rho}, \quad \eta=N^{01234}, \quad \theta=N^{01235} .
$$

This gives with (A.7) and (A.12)

$$
N^{a b c d e} L_{a b c d e}=\left(\begin{array}{cc}
0 & \mathbf{h}-\eta \mathbf{i}-\theta I_{4} \\
-\mathbf{h}+\eta \mathbf{i}-\theta I_{4} &
\end{array}\right) .
$$

We then get

$$
\begin{aligned}
& \Psi=\left(\begin{array}{ll}
\Psi_{l} & \Psi_{r} \\
\Psi_{g} & \Psi_{b}
\end{array}\right)= \\
& \left(\begin{array}{cc}
(\alpha+\omega) I_{4}+(\mathbf{b}+\mathbf{g})+(\mathbf{A}-\mathbf{i D})+\mathbf{i}(-\mathbf{c}+\mathbf{f})+(\zeta-\epsilon) \mathbf{i} & -(\beta+\theta) I_{4}+(\mathbf{a}+\mathbf{h})+(-\mathbf{B}+\mathbf{i C})+\mathbf{i}(-\mathbf{d}+\mathbf{e})+(\delta-\eta) \mathbf{i} \\
(\beta-\theta) I_{4}+(\mathbf{a}-\mathbf{h})+(\mathbf{B}+\mathbf{i C})+\mathbf{i}(\mathbf{d}+\mathbf{e})+(\delta+\eta) \mathbf{i} & (\alpha-\omega) I_{4}+(-\mathbf{b}+\mathbf{g})+(\mathbf{A}+\mathbf{i D})+\mathbf{i}(-\mathbf{c}-\mathbf{f})+(\zeta+\epsilon) \mathbf{i}
\end{array}\right)
\end{aligned}
$$

This implies

$$
\begin{aligned}
\Psi_{l} & =(\alpha+\omega)+(\mathbf{b}+\mathbf{g})+(\mathbf{A}-\mathbf{i D})+\mathbf{i}(-\mathbf{c}+\mathbf{f})+(\zeta-\epsilon) \mathbf{i} \\
\Psi_{r} & =-(\beta+\theta)+(\mathbf{a}+\mathbf{h})+(-\mathbf{B}+\mathbf{i} \mathbf{C})+\mathbf{i}(-\mathbf{d}+\mathbf{e})+(\delta-\eta) \mathbf{i} \\
\Psi_{g} & =(\beta-\theta)+(\mathbf{a}-\mathbf{h})+(\mathbf{B}+\mathbf{i C})+\mathbf{i}(\mathbf{d}+\mathbf{e})+(\delta+\eta) \mathbf{i} \\
\Psi_{b} & =(\alpha-\omega)+(-\mathbf{b}+\mathbf{g})+(\mathbf{A}+\mathbf{i D})+\mathbf{i}(-\mathbf{c}-\mathbf{f})+(\zeta+\epsilon) \mathbf{i} .
\end{aligned}
$$

In $\mathrm{Cl}_{1,3}$ the reverse of

$$
A=\langle A\rangle_{0}+\langle A\rangle_{1}+\langle A\rangle_{2}+\langle A\rangle_{3}+\langle A\rangle_{4}
$$

is

$$
\tilde{A}=\langle A\rangle_{0}+\langle A\rangle_{1}-\langle A\rangle_{2}-\langle A\rangle_{3}+\langle A\rangle_{4}
$$

we must change the sign of bivectors $\mathbf{A}, \mathbf{B}, \mathbf{i C}, \mathbf{i D}$, and trivectors ic, id, ie, if and we then get

$$
\begin{aligned}
& \tilde{\Psi}_{l}=(\alpha+\omega)+(\mathbf{b}+\mathbf{g})+(-\mathbf{A}+\mathbf{i D})+\mathbf{i}(\mathbf{c}-\mathbf{f})+(\zeta-\epsilon) \mathbf{i} \\
& \tilde{\Psi}_{r}=-(\beta+\theta)+(\mathbf{a}+\mathbf{h})+(\mathbf{B}-\mathbf{i} \mathbf{C})+\mathbf{i}(\mathbf{d}-\mathbf{e})+(\delta-\eta) \mathbf{i} \\
& \tilde{\Psi}_{g}=(\beta-\theta)+(\mathbf{a}-\mathbf{h})-(\mathbf{B}+\mathbf{i} \mathbf{C})-\mathbf{i}(\mathbf{d}+\mathbf{e})+(\delta+\eta) \mathbf{i} \\
& \tilde{\Psi}_{b}=(\alpha-\omega)+(-\mathbf{b}+\mathbf{g})-(\mathbf{A}+\mathbf{i D})+\mathbf{i}(\mathbf{c}+\mathbf{f})+(\zeta+\epsilon) \mathbf{i} .
\end{aligned}
$$

The reverse, in $C_{1,5}$ now, of

$$
A=\langle A\rangle_{0}+\langle A\rangle_{1}+\langle A\rangle_{2}+\langle A\rangle_{3}+\langle A\rangle_{4}+\langle A\rangle_{5}+\langle A\rangle_{6}
$$

is

$$
\tilde{A}=\langle A\rangle_{0}+\langle A\rangle_{1}-\langle A\rangle_{2}-\langle A\rangle_{3}+\langle A\rangle_{4}+\langle A\rangle_{5}-\langle A\rangle_{6}
$$

Only terms which change sign, with (A.13), (A.18) and (A.20), are scalars $\epsilon$ and $\omega$, vectors b, c, d, e and bivectors $\mathbf{A}, \mathbf{B}, \mathbf{C}$. These changes of sign are not the same in $C l_{1,5}$ as in $C l_{1,3}$. Differences are corrected by the fact that the reversion in $C l_{1,5}$ also exchanges the place of $\Psi_{l}$ and $\Psi_{b}$ terms. We then get from (A.25)

$$
\begin{aligned}
& \tilde{\Psi}= \\
& \left(\begin{array}{cc}
(\alpha-\omega) I_{4}+(-\mathbf{b}+\mathbf{g})+(-\mathbf{A}-\mathbf{i D})+\mathbf{i}(\mathbf{c}+\mathbf{f})+(\zeta+\epsilon) \mathbf{i} & -(\beta+\theta) I_{4}+(\mathbf{a}+\mathbf{h})+(\mathbf{B}-\mathbf{i C})+\mathbf{i}(\mathbf{d}-\mathbf{e})+(\delta-\eta) \mathbf{i} \\
(\beta-\theta) I_{4}+(\mathbf{a}-\mathbf{h})-(\mathbf{B}+\mathbf{i C})-\mathbf{i}(\mathbf{d}+\mathbf{e})+(\delta+\eta) \mathbf{i} & (\alpha+\omega) I_{4}+(\mathbf{b}+\mathbf{g})+(-\mathbf{A}+\mathbf{i D})+\mathbf{i}(\mathbf{c}-\mathbf{f})+(\zeta-\epsilon) \mathbf{i}
\end{array}\right) \\
& \quad=\left(\begin{array}{ll}
\tilde{\Psi}_{b} & \tilde{\Psi}_{r} \\
\tilde{\Psi}_{g} & \tilde{\Psi}_{l}
\end{array}\right)
\end{aligned}
$$


This link between the reversion in $\mathrm{Cl}_{1,3}$ and the reversion in $\mathrm{Cl}_{1,5}$ is necessary to get an invariant wave equation. It is not general, for instance the reversion in $\mathrm{Cl}_{3}$ is not linked to the reversion in $\mathrm{Cl}_{2,3}$.

\section{Appendix B. Scalar Densities and $\chi$ Terms}

There are $6 \times 5 / 2=15$ such complex scalar densities:

$$
\begin{aligned}
& s_{1}=2\left(\xi_{1 \overline{u r}} \eta_{1 \text { ug }}^{*}+\xi_{2 \overline{\text { ur }}} \eta_{2 u g}^{*}\right)=2\left(\eta_{2 u r}^{*} \eta_{1 u g}^{*}-\eta_{\text {lur }}^{*} \eta_{2 u g}^{*}\right) \\
& s_{2}=2\left(\xi_{1 \overline{u g}} \eta_{1 u b}^{*}+\xi_{2 \overline{u g}} \eta_{2 u b}^{*}\right)=2\left(\eta_{2 u g}^{*} \eta_{1 u b}^{*}-\eta_{1 u g}^{*} \eta_{2 u b}^{*}\right) \\
& s_{3}=-2\left(\xi_{\bar{u} \bar{r}} \eta_{1 u b}^{*}+\xi_{2 \bar{u} r} \eta_{2 u b}^{*}\right)=2\left(\eta_{2 u b}^{*} \eta_{1 u r}^{*}-\eta_{1 u b}^{*} \eta_{2 u r}^{*}\right) \\
& s_{4}=2\left(\xi_{1 \overline{d r}} \eta_{1 d g}^{*}+\xi_{2 \overline{d r}} \eta_{2 d g}^{*}\right)=2\left(\eta_{2 d r}^{*} \eta_{1 d g}^{*}-\eta_{1 d r}^{*} \eta_{2 d g}^{*}\right) \\
& s_{5}=2\left(\xi_{1 \bar{d} g} \eta_{1 d b}^{*}+\xi_{2 \bar{d} g} \eta_{2 d b}^{*}\right)=2\left(\eta_{2 d g}^{*} \eta_{1 d b}^{*}-\eta_{1 d g}^{*} \eta_{2 d b}^{*}\right) \\
& s_{6}=-2\left(\xi_{1 \overline{d r}} \eta_{1 d b}^{*}+\xi_{2 \overline{d r}} \eta_{2 d b}^{*}\right)=2\left(\eta_{2 d b}^{*} \eta_{1 d r}^{*}-\eta_{1 d b}^{*} \eta_{2 d r}^{*}\right) \\
& s_{7}=2\left(\xi_{1 \bar{r} r} \eta_{1 d r}^{*}+\xi_{2 \bar{r} r} \eta_{2 d r}^{*}\right)=2\left(\eta_{2 u r}^{*} \eta_{1 d r}^{*}-\eta_{1 u r}^{*} \eta_{2 d r}^{*}\right) \\
& s_{8}=2\left(\xi_{1 \overline{u g}} \eta_{1 d g}^{*}+\xi_{2 \overline{u g}} \eta_{2 d g}^{*}\right)=2\left(\eta_{2 u g}^{*} \eta_{1 d g}^{*}-\eta_{1 u g}^{*} \eta_{2 d g}^{*}\right) \\
& s_{9}=2\left(\xi_{1 \overrightarrow{ }} \eta_{1 d b}^{*}+\xi_{2 \bar{b}} \eta_{2 d b}^{*}\right)=2\left(\eta_{2 u b}^{*} \eta_{1 d b}^{*}-\eta_{1 u b}^{*} \eta_{2 d b}^{*}\right) \\
& s_{10}=2\left(\xi_{1 \overline{u r}} \eta_{1 d g}^{*}+\xi_{2 \overline{u r}} \eta_{2 d g}^{*}\right)=2\left(\eta_{2 u r}^{*} \eta_{1 d g}^{*}-\eta_{1 u r}^{*} \eta_{2 d g}^{*}\right) \\
& s_{11}=2\left(\xi_{1 \overline{u g}} \eta_{1 d b}^{*}+\xi_{2 \bar{u} g} \eta_{2 d b}^{*}\right)=2\left(\eta_{2 u g}^{*} \eta_{1 d b}^{*}-\eta_{1 u g}^{*} \eta_{2 d b}^{*}\right) \\
& s_{12}=-2\left(\xi_{1 \overline{d r}} \eta_{1 u b}^{*}+\xi_{2 \overline{d r}} \eta_{2 u b}^{*}\right)=2\left(\eta_{2 u b}^{*} \eta_{1 d r}^{*}-\eta_{1 u b}^{*} \eta_{2 d r}^{*}\right) \\
& s_{13}=2\left(\xi_{1 \overline{u r}} \eta_{1 d b}^{*}+\xi_{2 \overline{u r}} \eta_{2 d b}^{*}\right)=2\left(\eta_{2 u r}^{*} \eta_{1 d b}^{*}-\eta_{1 u r}^{*} \eta_{2 d b}^{*}\right) \\
& s_{14}=-2\left(\xi_{1 \overline{d r}} \eta_{1 u g}^{*}+\xi_{2 \overline{d r}} \eta_{2 u g}^{*}\right)=2\left(\eta_{2 u g}^{*} \eta_{1 d r}^{*}-\eta_{1 u g}^{*} \eta_{2 d r}^{*}\right) \\
& s_{15}=-2\left(\xi_{1 \bar{d} g} \eta_{1 u b}^{*}+\xi_{2 \bar{d} g} \eta_{2 u b}^{*}\right)=2\left(\eta_{2 u b}^{*} \eta_{1 d g}^{*}-\eta_{1 u b}^{*} \eta_{2 d g}^{*}\right) \text {. }
\end{aligned}
$$

We used in [2]

$$
\chi_{l}=\frac{1}{\rho_{1}^{2}}\left(\begin{array}{cc}
a_{1}^{*} \phi_{e}+a_{2}^{*} \phi_{n} \sigma_{1}+a_{3}^{*} \phi_{n} & -a_{2}^{*} \phi_{e L} \sigma_{1}+a_{3}^{*} \phi_{e R} \\
a_{2} \hat{\phi}_{e L} \sigma_{1}+a_{3} \hat{\phi}_{e R} & a_{1} \hat{\phi}_{e}-a_{2} \hat{\phi}_{n} \sigma_{1}+a_{3} \hat{\phi}_{n}
\end{array}\right)
$$

with $\phi_{e R}=\phi_{e}\left(1+\sigma_{3}\right) / 2$ and $\phi_{e L}=\phi_{e}\left(1-\sigma_{3}\right) / 2$, and we need now

$$
\begin{aligned}
& \rho_{2}^{2} \chi_{r}=\left(\begin{array}{ll}
\left(s_{4}^{*} \phi_{d g}-s_{6}^{*} \phi_{d b}-s_{7}^{*} \phi_{u r}-s_{12}^{*} \phi_{u b}-s_{14}^{*} \phi_{u g}\right) \sigma_{1} & \left(s_{1}^{*} \phi_{u g}-s_{3}^{*} \phi_{u b}+s_{7}^{*} \phi_{d r}+s_{10}^{*} \phi_{d g}+s_{13}^{*} \phi_{d b}\right) \sigma_{1} \\
\left(-s_{1} \hat{\phi}_{u g}+s_{3} \hat{\phi}_{u b}-s_{7} \hat{\phi}_{d r}-s_{10} \hat{\phi}_{d g}-s_{13} \hat{\phi}_{d b}\right) \sigma_{1} & \left(-s_{4} \hat{\phi}_{d g}+s_{6} \hat{\phi}_{d b}+s_{7} \hat{\phi}_{u r}+s_{12} \hat{\phi}_{u b}+s_{14} \hat{\phi}_{u g}\right) \sigma_{1}
\end{array}\right) \\
& \rho_{2}^{2} \chi_{g}=\left(\begin{array}{ll}
\left(s_{5}^{*} \phi_{d b}-s_{4}^{*} \phi_{d r}-s_{8}^{*} \phi_{u g}-s_{10}^{*} \phi_{u r}-s_{15}^{*} \phi_{u b}\right) \sigma_{1} & \left(s_{2}^{*} \phi_{u b}-s_{1}^{*} \phi_{u r}+s_{8}^{*} \phi_{d g}+s_{11}^{*} \phi_{d b}+s_{14}^{*} \phi_{d r}\right) \sigma_{1} \\
\left(-s_{2} \hat{\phi}_{u b}+s_{1} \hat{\phi}_{u r}-s_{8} \hat{\phi}_{d g}-s_{11} \hat{\phi}_{d b}-s_{14} \hat{\phi}_{d r}\right) \sigma_{1} & \left(-s_{5} \hat{\phi}_{d b}+s_{4} \hat{\phi}_{d r}+s_{8} \hat{\phi}_{u g}+s_{10} \hat{\phi}_{u r}+s_{15} \hat{\phi}_{u b}\right) \sigma_{1}
\end{array}\right) \\
& \rho_{2}^{2} \chi_{b}=\left(\begin{array}{ll}
\left(s_{6}^{*} \phi_{d r}-s_{5}^{*} \phi_{d g}-s_{9}^{*} \phi_{u b}-s_{11}^{*} \phi_{u g}-s_{13}^{*} \phi_{u r}\right) \sigma_{1} & \left(s_{3}^{*} \phi_{u r}-s_{2}^{*} \phi_{u g}+s_{9}^{*} \phi_{d b}+s_{12}^{*} \phi_{d r}+s_{15}^{*} \phi_{d g}\right) \sigma_{1} \\
\left(-s_{3} \hat{\phi}_{u r}+s_{2} \hat{\phi}_{u g}-s_{9} \hat{\phi}_{d b}-s_{12} \hat{\phi}_{d r}-s_{15} \hat{\phi}_{d g}\right) \sigma_{1} & \left(-s_{6} \hat{\phi}_{d r}+s_{5} \hat{\phi}_{d g}+s_{9} \hat{\phi}_{u b}+s_{11} \hat{\phi}_{u g}+s_{13} \hat{\phi}_{u r}\right) \sigma_{1}
\end{array}\right) .
\end{aligned}
$$




\section{Appendix C. Gauge Invariance, Details}

C.1. Gauge Group Generated by $\mathrm{i}_{3}$

We name $f_{3}$ the gauge transformation

$$
f_{3}: \Psi^{c} \mapsto \underline{\mathbf{i}} \Gamma_{3}\left(\Psi^{c}\right)=\left(\begin{array}{cc}
0 & \mathbf{i} \Psi_{r} \\
-\mathbf{i} \Psi_{g} & 0
\end{array}\right)
$$

which implies

$$
\begin{aligned}
& {\left[\exp \left(\theta f_{3}\right)\right]\left(\Psi^{c}\right)=\left(\begin{array}{cc}
0 & \mathrm{e}^{\theta \mathbf{i}} \Psi_{r} \\
\mathrm{e}^{-\theta \mathbf{i}} \Psi_{g} & \Psi_{b}
\end{array}\right)=\left(\begin{array}{cc}
0 & \Psi_{r}^{\prime} \\
\Psi_{g}^{\prime} & \Psi_{b}^{\prime}
\end{array}\right)} \\
& \Psi_{r}^{\prime}=\mathrm{e}^{\theta \mathbf{i}} \Psi_{r} \\
& \Psi_{g}^{\prime}=\mathrm{e}^{-\theta \mathrm{i}} \Psi_{r} \\
& \Psi_{b}^{\prime}=\Psi_{b} .
\end{aligned}
$$

The equality (C.3) is equivalent to

$$
\left(\begin{array}{cc}
\phi_{d r}^{\prime} & \phi_{u r}^{\prime} \\
\hat{\phi}_{u r}^{\prime} & \hat{\phi}_{d r}^{\prime}
\end{array}\right)=\left(\begin{array}{cc}
\mathrm{e}^{i \theta} & 0 \\
0 & \mathrm{e}^{-i \theta}
\end{array}\right)\left(\begin{array}{cc}
\phi_{d r} & \phi_{u r} \\
\hat{\phi}_{u r} & \hat{\phi}_{d r}
\end{array}\right) .
$$

The equality (C.4) is equivalent to

$$
\left(\begin{array}{ll}
\phi_{d g}^{\prime} & \phi_{u g}^{\prime} \\
\hat{\phi}_{u g}^{\prime} & \hat{\phi}_{d g}^{\prime}
\end{array}\right)=\left(\begin{array}{cc}
\mathrm{e}^{-i \theta} & 0 \\
0 & \mathrm{e}^{i \theta}
\end{array}\right)\left(\begin{array}{ll}
\phi_{d g} & \phi_{u g} \\
\hat{\phi}_{u g} & \hat{\phi}_{d g}
\end{array}\right) .
$$

We get

$$
\begin{array}{ll}
\eta_{1 d r}^{\prime *}=\mathrm{e}^{-i \theta} \eta_{1 d r}^{*}, & \eta_{1 u r}^{\prime *}=\mathrm{e}^{-i \theta} \eta_{1 u r}^{*} \\
\eta_{2 d r}^{\prime *}=\mathrm{e}^{-i \theta} \eta_{2 d r}^{*}, & \eta_{2 u r}^{\prime *}=\mathrm{e}^{-i \theta} \eta_{2 u r}^{*} \\
\eta_{1 d g}^{\prime *}=\mathrm{e}^{i \theta} \eta_{1 d g}^{*}, & \eta_{1 u g}^{\prime *}=\mathrm{e}^{\mathrm{i} \theta} \eta_{1 u g}^{*} \\
\eta_{2 d g}^{\prime *}=\mathrm{e}^{i \theta} \eta_{2 d g}^{*}, & \eta_{2 u g}^{\prime *}=\mathrm{e}^{i \theta} \eta_{2 u g}^{*} .
\end{array}
$$

This gives

from which we get

$$
\begin{aligned}
& s_{1}^{\prime}=s_{1}, \quad s_{2}^{\prime}=\mathrm{e}^{-i \theta} s_{2}, \quad s_{3}^{\prime}=\mathrm{e}^{i \theta} s_{3} \\
& s_{4}^{\prime}=s_{4}, \quad s_{5}^{\prime}=\mathrm{e}^{-i \theta} s_{5}, \quad s_{6}^{\prime}=\mathrm{e}^{i \theta} s_{6} \\
& s_{9}^{\prime}=s_{9}, \quad s_{8}^{\prime}=\mathrm{e}^{-2 i \theta} s_{8}, \quad s_{7}^{\prime}=\mathrm{e}^{2 i \theta} s_{7} \\
& s_{10}^{\prime}=s_{10}, \quad s_{11}^{\prime}=\mathrm{e}^{-i \theta} s_{11}, \quad s_{12}^{\prime}=\mathrm{e}^{i \theta} s_{12} \\
& s_{14}^{\prime}=s_{14}, \quad s_{15}^{\prime}=\mathrm{e}^{-i \theta} s_{15}, \quad s_{13}^{\prime}=\mathrm{e}^{i \theta} s_{13}
\end{aligned}
$$

$$
\begin{gathered}
s_{j}^{\prime} s_{j}^{* *}=s_{j} s_{j}^{*}, \quad j=1,2, \cdots, 15 \\
\rho^{\prime}=\rho \\
\chi_{r}^{\prime}=\mathrm{e}^{-\mathrm{i} \theta} \chi_{r}
\end{gathered}
$$




$$
\chi_{g}^{\prime}=\mathrm{e}^{\mathrm{i} \theta} \chi_{g} .
$$

These relations are the awaited ones because

$$
\begin{aligned}
\partial \Psi_{r}^{\prime} & =\partial\left(\mathrm{e}^{\mathrm{i} \theta} \Psi_{r}\right)=\mathrm{e}^{-\mathrm{i} \theta}\left(-\mathrm{i} \partial \theta \Psi_{r}+\partial \Psi_{r}\right) \\
\partial \Psi_{g}^{\prime} & =\partial\left(\mathrm{e}^{-\mathrm{i} \theta} \Psi_{g}\right)=\mathrm{e}^{\mathrm{i} \theta}\left(\mathbf{i} \partial \theta \Psi_{g}+\partial \Psi_{g}\right) \\
\mathbf{G}^{\prime 3} & =\mathbf{G}^{3}-\frac{2}{g_{3}} \partial \theta .
\end{aligned}
$$

\section{C.2. Gauge Group Generated by $\underline{\mathrm{i}} \Gamma_{8}$}

We name $f_{8}$ the gauge transformation

$$
f_{8}: \Psi^{c} \mapsto \underline{\mathbf{i}} \Gamma_{8}\left(\Psi^{c}\right)=\left(\begin{array}{cc}
0 & \frac{\mathbf{i}}{\sqrt{3}} \Psi_{r} \\
\frac{\mathbf{i}}{\sqrt{3}} \Psi_{g} & -\frac{2 \mathbf{i}}{\sqrt{3}} \Psi_{b}
\end{array}\right)
$$

which implies

$$
\begin{aligned}
& {\left[\exp \left(\theta f_{8}\right)\right]\left(\Psi^{c}\right)=\left(\begin{array}{cc}
0 & \mathrm{e}^{\frac{\theta \mathbf{i}}{\sqrt{3}}} \Psi_{r} \\
\frac{\theta \mathbf{i}}{\sqrt{3}} \Psi_{g} & \mathrm{e}^{-\frac{2 \theta \mathbf{i}}{\sqrt{3}}} \Psi_{b}
\end{array}\right)=\left(\begin{array}{cc}
0 & \Psi_{r}^{\prime} \\
\Psi_{g}^{\prime} & \Psi_{b}^{\prime}
\end{array}\right)} \\
& \Psi_{r}^{\prime}=\exp \left(\frac{\theta \mathbf{i}}{\sqrt{3}}\right) \Psi_{r} \\
& \Psi_{g}^{\prime}=\exp \left(\frac{\theta \mathbf{i}}{\sqrt{3}}\right) \Psi_{g} \\
& \Psi_{b}^{\prime}=\exp \left(-\frac{2 \theta \mathbf{i}}{\sqrt{3}}\right) \Psi_{b} .
\end{aligned}
$$

This gives

$$
\begin{aligned}
& \phi_{d r}^{\prime}=\exp \left(\frac{i \theta}{\sqrt{3}}\right) \phi_{d r}, \quad \phi_{u r}^{\prime}=\exp \left(\frac{i \theta}{\sqrt{3}}\right) \phi_{u r} \\
& \phi_{d g}^{\prime}=\exp \left(\frac{i \theta}{\sqrt{3}}\right) \phi_{d g}, \quad \phi_{u g}^{\prime}=\exp \left(\frac{i \theta}{\sqrt{3}}\right) \phi_{u g} \\
& \phi_{d b}^{\prime}=\exp \left(-\frac{2 i \theta}{\sqrt{3}}\right) \phi_{d b}, \quad \phi_{u b}^{\prime}=\exp \left(-\frac{2 i \theta}{\sqrt{3}}\right) \phi_{u b} .
\end{aligned}
$$

We then get

$$
\begin{array}{ll}
\eta_{1 d r}^{\prime *}=\exp \left(\frac{i \theta}{\sqrt{3}}\right) \eta_{1 d r}^{*}, \quad \eta_{1 d g}^{\prime *}=\exp \left(\frac{i \theta}{\sqrt{3}}\right) \eta_{1 d g}^{*}, \quad \eta_{1 d b}^{\prime *}=\exp \left(-\frac{2 i \theta}{\sqrt{3}}\right) \eta_{1 d g}^{*} \\
\eta_{2 d r}^{\prime *}=\exp \left(\frac{i \theta}{\sqrt{3}}\right) \eta_{2 d r}^{*}, \quad \eta_{2 d g}^{\prime *}=\exp \left(\frac{i \theta}{\sqrt{3}}\right) \eta_{2 d g}^{*}, \quad \eta_{2 d b}^{\prime *}=\exp \left(-\frac{2 i \theta}{\sqrt{3}}\right) \eta_{2 d g}^{*} \\
\eta_{1 u r}^{\prime *}=\exp \left(\frac{i \theta}{\sqrt{3}}\right) \eta_{1 u r}^{*}, \quad \eta_{1 u g}^{\prime *}=\exp \left(\frac{i \theta}{\sqrt{3}}\right) \eta_{1 u g}^{*}, \quad \eta_{1 u b}^{\prime *}=\exp \left(-\frac{2 i \theta}{\sqrt{3}}\right) \eta_{1 u g}^{*}
\end{array}
$$




$$
\eta_{2 u r}^{\prime *}=\exp \left(\frac{i \theta}{\sqrt{3}}\right) \eta_{2 u r}^{*}, \quad \eta_{2 u g}^{\prime *}=\exp \left(\frac{i \theta}{\sqrt{3}}\right) \eta_{2 u g}^{*}, \quad \eta_{2 u b}^{\prime *}=\exp \left(-\frac{2 i \theta}{\sqrt{3}}\right) \eta_{2 u g}^{*}
$$

This implies

$$
\begin{aligned}
& s_{1}^{\prime}=\exp \left(\frac{2 i \theta}{\sqrt{3}}\right) s_{1}, \quad s_{2}^{\prime}=\exp \left(-\frac{i \theta}{\sqrt{3}}\right) s_{2}, \quad s_{3}^{\prime}=\exp \left(-\frac{i \theta}{\sqrt{3}}\right) s_{3} \\
& s_{4}^{\prime}=\exp \left(\frac{2 i \theta}{\sqrt{3}}\right) s_{4}, \quad s_{5}^{\prime}=\exp \left(-\frac{i \theta}{\sqrt{3}}\right) s_{5}, \quad s_{6}^{\prime}=\exp \left(-\frac{i \theta}{\sqrt{3}}\right) s_{6} \\
& s_{7}^{\prime}=\exp \left(\frac{2 i \theta}{\sqrt{3}}\right) s_{7}, \quad s_{8}^{\prime}=\exp \left(\frac{2 i \theta}{\sqrt{3}}\right) s_{8}, \quad s_{9}^{\prime}=\exp \left(-\frac{4 i \theta}{\sqrt{3}}\right) s_{9} \\
& s_{10}^{\prime}=\exp \left(\frac{2 i \theta}{\sqrt{3}}\right) s_{10}, \quad s_{11}^{\prime}=\exp \left(-\frac{i \theta}{\sqrt{3}}\right) s_{11}, \quad s_{12}^{\prime}=\exp \left(-\frac{i \theta}{\sqrt{3}}\right) s_{12} \\
& s_{13}^{\prime}=\exp \left(-\frac{i \theta}{\sqrt{3}}\right) s_{13}, \quad s_{14}^{\prime}=\exp \left(\frac{2 i \theta}{\sqrt{3}}\right) s_{14}, \quad s_{15}^{\prime}=\exp \left(-\frac{i \theta}{\sqrt{3}}\right) s_{15} .
\end{aligned}
$$

We then get the awaited results

$$
\begin{aligned}
& s_{j}^{\prime} s_{j}^{\prime *}=s_{j} s_{j}^{*}, \quad j=1,2, \cdots, 15, \quad \rho^{\prime}=\rho \\
& \chi_{r}^{\prime}=\exp \left(-\frac{\mathbf{i} \theta}{\sqrt{3}}\right) \chi_{r}, \quad \chi_{g}^{\prime}=\exp \left(-\frac{\mathbf{i} \theta}{\sqrt{3}}\right) \chi_{g}, \quad \chi_{b}^{\prime}=\exp \left(\frac{2 \mathbf{i} \theta}{\sqrt{3}}\right) \chi_{b} .
\end{aligned}
$$


Scientific Research Publishing (SCIRP) is one of the largest Open Access journal publishers. It is currently publishing more than 200 open access, online, peer-reviewed journals covering a wide range of academic disciplines. SCIRP serves the worldwide academic communities and contributes to the progress and application of science with its publication.

Other selected journals from SCIRP are listed as below. Submit your manuscript to us via either submit@scirp.org or Online Submission Portal.
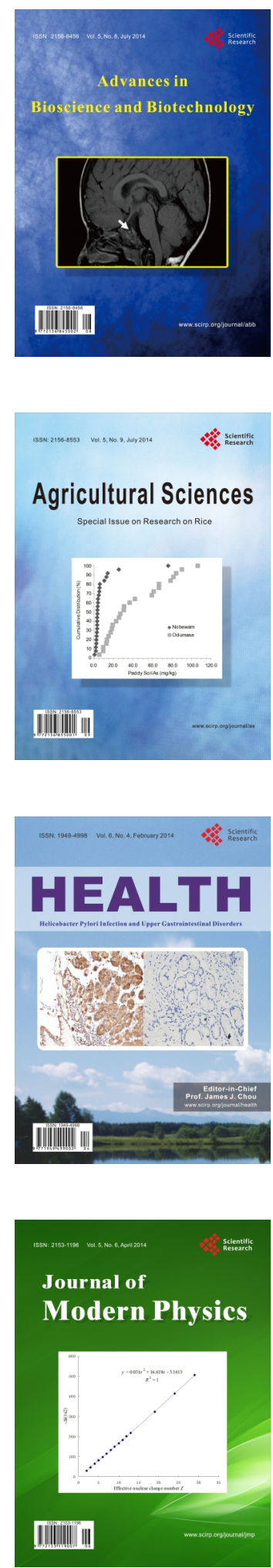
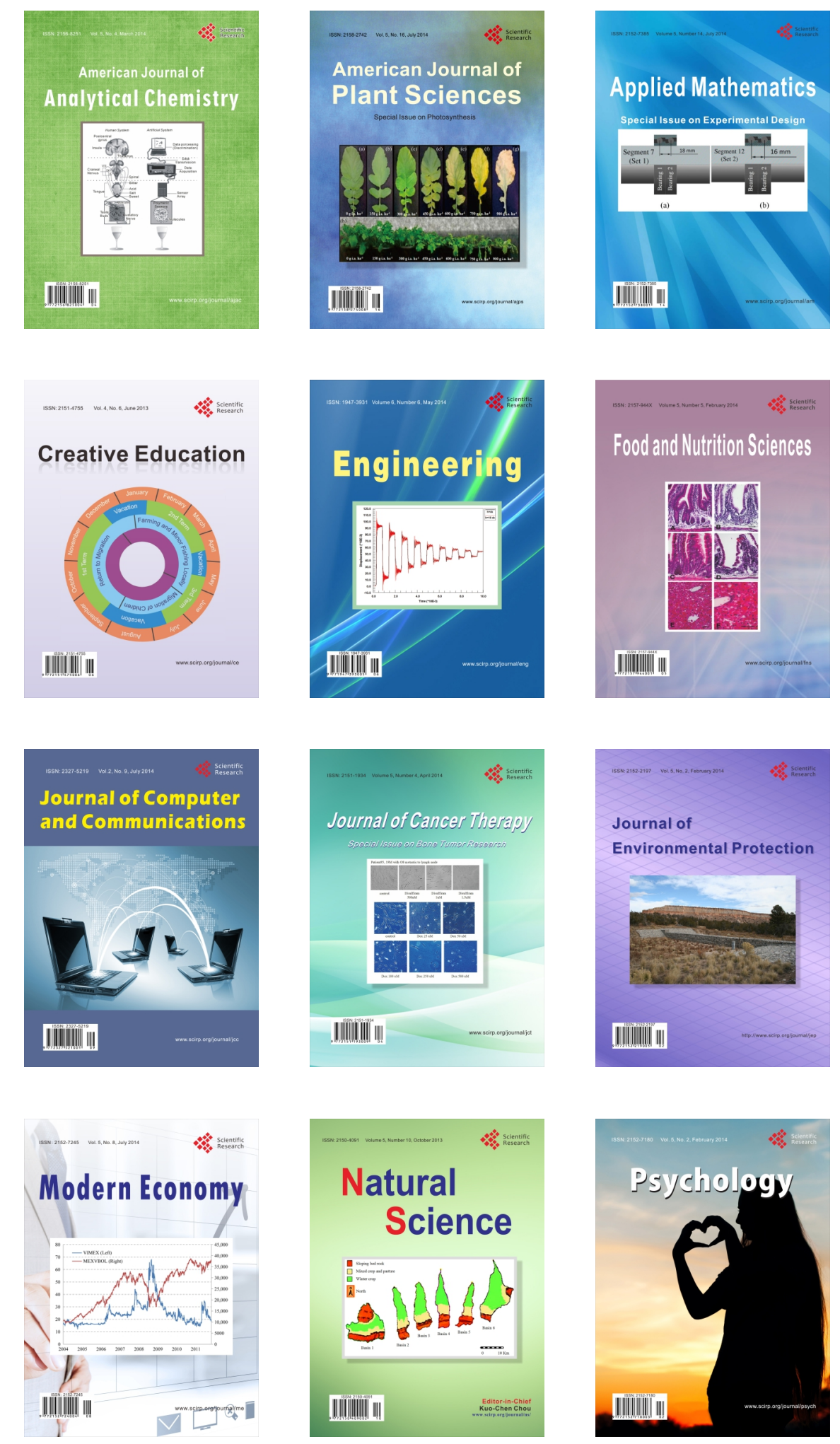